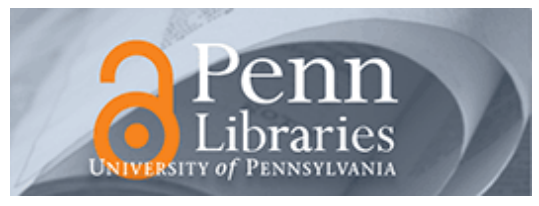

University of Pennsylvania

ScholarlyCommons

Management Papers

Wharton Faculty Research

$5-2008$

\title{
The Dual Role of Modularity: Innovation and Imitation
}

Sendil K. Ethraj

Daniel A. Levinthal

University of Pennsylvania

Rishi R. Roy

Follow this and additional works at: https://repository.upenn.edu/mgmt_papers

Part of the Business Administration, Management, and Operations Commons

\section{Recommended Citation}

Ethraj, S. K., Levinthal, D. A., \& Roy, R. R. (2008). The Dual Role of Modularity: Innovation and Imitation. Management Science, 54 (5), 939-955. http://dx.doi.org/10.1287/mnsc.1070.0775

This paper is posted at ScholarlyCommons. https://repository.upenn.edu/mgmt_papers/137

For more information, please contact repository@pobox.upenn.edu. 


\title{
The Dual Role of Modularity: Innovation and Imitation
}

\begin{abstract}
Modularity has been heralded as an organizational and technical architecture that enhances incremental and modular innovation. Less attention has been paid to the possible implications of modular architectures for imitation. To understand the implications of modular designs for competitive advantage, one must consider the dual impact of modularity on innovation and imitation jointly. In an attempt to do so, we set up three alternative structures that vary in the extent of modularity and hence in the extent of design complexity: nonmodular, modular, and nearly modular designs. In each structure, we examine the trade-offs between innovation benefits and imitation deterrence. The results of our computational experiments indicate that modularization enables performance gains through innovation but, at the same time, sets the stage for those gains to be eroded through imitation. In contrast, performance differences between the leaders and imitators persist in the nearly modular and the nonmodular structures. Overall, we find that design complexity poses a significant trade-off between innovation benefits (i.e., generating superior strategies that create performance differences) and imitation deterrence (i.e., preserving the performance differences). We also examine the robustness of our results to variations in imitation accuracy. In addition to documenting the overall robustness of our principal finding, the ancillary analyses provide a more nuanced rendering of the relationship between the architecture of complexity and imitation efforts.
\end{abstract}

\section{Keywords}

modularity, complex systems, imitation

Disciplines

Business Administration, Management, and Operations 
The Dual Role of Modularity: Innovation and Imitation*

\author{
SENDIL K. ETHIRAJ \\ Stephen M. Ross School of Business \\ 701 Tappan Ave., ER4608 \\ University of Michigan, Ann Arbor, MI 48109 \\ Ph. 734-764-1230 \\ E-mail: sendil@umich.edu \\ DANIEL LEVINTHAL \\ The Wharton School of Business \\ 3620 Locust Walk, Suite 2000 \\ University of Pennsylvania, Philadelphia PA 19104 \\ Ph. 215-898-6826 \\ E-mail: levinthal@wharton.upenn.edu \\ and \\ RISHI R. ROY \\ McKinsey and Co. Inc \\ 55 E. 52nd Street, 21st Floor \\ New York, NY 10055 \\ E-mail: rishi_roy@mckinsey.com
}

Dated: March 2007

\footnotetext{
${ }^{*}$ We thank Kathy Eisenhardt for comments on an earlier paper and John Paul Macduffie for pointing us to empirical examples in the Auto Industry, both of which sowed the seeds for this paper. We owe a special thanks to the Associate Editor and the two anonymous reviewers who provided extraordinarily detailed and informed set of comments that improved the quality of this paper. We also thank Carliss Baldwin for her thoughtful and provocative feedback on this research. The first author thanks the Michael R. and Mary Kay Hallman fellowship at the University of Michigan for financial support. We are solely responsible for all errors and omissions.
} 


\section{Management Insight Statement}

What makes competitive advantage enduring and prevents its imitation is a central question in strategy research. In this paper, we investigate how and why complexity deters imitation efforts. We argue that design complexity captures the ease of making localized changes without affecting the whole organization. Based on this idea of complexity we identify three organizational structural types: fully modular, nearly modular and non-modular. The three designs differ in the extent to which they encapsulate interdependencies. We show that the potential for incremental innovation in an organization increases when one moves from non-modular to modular structures. In contrast, the potential for an organization to deter imitation decreases from non-modular to modular structures. We elaborate how and why design complexity affects the nature of the trade-off between innovation and imitation deterrence and thereby help address questions about the effective allocation of imitation "energies," either for imitating firms to increase the efficacy of their imitation efforts or for innovating firms to effectively deter imitation. We discuss how our analyses shed light on several contemporary examples of innovation and imitation deterrence, such as the emergence of low-cost airlines and the rise of IBM in the mainframe computer industry. 


\title{
The Dual Role of Modularity: Innovation and Imitation
}

\begin{abstract}
Modularity has been heralded as an organizational and technical architecture that enhances incremental and modular innovation. Less attention has been paid to the possible implications of modular architectures for imitation. To understand the implications of modular designs for competitive advantage, one must consider the dual impact of modularity on innovation and imitation jointly. In an attempt to do so, we set up three alternative structures that vary in the extent of modularity and hence in the extent of design complexity: non-modular, modular, and nearly modular designs. In each structure, we examine the trade-offs between innovation benefits and imitation deterrence. The results of our computational experiments indicate that modularization enables performance gains through innovation but, at the same time, sets the stage for those gains to be eroded through imitation. In contrast, performance differences between the leaders and imitators persist in the nearly modular and the non-modular structures. Overall, we find that design complexity poses a significant trade-off between innovation benefits (i.e., generating superior strategies that create performance differences) and imitation deterrence (i.e., preserving the performance differences). We also examine the robustness of our results to variations in imitation accuracy. In addition to documenting the overall robustness of our principal finding, the ancillary analyses provide a more nuanced rendering of the relationship between the architecture of complexity and imitation efforts.
\end{abstract}




\section{Introduction}

How do firms create or acquire capabilities or positions that generate competitive advantage (Porter 1980; Rumelt 1984; Winter 1995)? What are the mechanisms available to protect them from being imitated or substituted (Rumelt 1984; Reed and DeFillippi 1990)? These are perhaps the two central questions guiding strategy research. While a significant volume of research has examined the nature of capabilities and positions and the conditions under which they generate competitive advantage, there is comparatively less work on barriers to imitation or substitution. The existing work addressing the latter question, mostly conceptual in nature, suggests that imitation or substitution barriers are largely a function of complexity in executed strategies (Rumelt 1984; Barney 1991). More recently, Rivkin (2000; 2001), using a formal computational model, demonstrated how greater complexity of strategies deters imitation efforts. In this paper we seek to increase our understanding of the joint effects of complexity on innovation and imitation deterrence.

In addressing this question, we link the issues of capability creation and imitation, respectively, by exploiting an important but under-explored link to research on modularity and complexity. Simon (1962), in his work on the architecture of complexity, argued that systems that are hierarchical and nearly decomposable help reduce the complexity of the design challenge. The twin principles of hierarchy and near-decomposability now form the cornerstones of modular designs (Parnas 1972; Baldwin and Clark 2000). It is generally accepted that a modular design is based on a principle of encapsulating interdependencies within self-contained units called modules and minimizing reciprocal interdependencies between modules. Encapsulating interdependencies makes a system nearly decomposable, and minimizing reciprocal interdependencies makes it hierarchical. As a result, modular structures more easily allow incremental and localized innovation within modules and thus help reduce design complexity. Conversely, in non-modular (or integral) structures, the management of interdependencies is not the primary guiding principle 
of design (see Ulrich 1995 for a discussion of the costs and benefits of modular and integral designs). As systems grow larger, the disruptive impact of interdependencies on design grows greater than proportionately. Other things held constant, for systems of identical size the complexity of an integral design will be significantly greater than the complexity of a modular one. Therefore, holding size constant, the degree of modularity should provide important insight into the fundamental question of how and why complexity deters imitation.

We aim to examine the relationship between complexity and imitation deterrence using several controlled experiments in a computational model (see Ethiraj and Levinthal 2004b which employs a similar model to explore the trade-offs between coarser and finer partitioning of modules). In the first experiment, holding the overall level of interdependence in the firm constant, we set up three alternative structures that vary in the extent of design complexity: (1) perfectly modular structure with no interdependencies between modules, (2) nearly modular structure with minimal interdependencies between modules, and (3) non-modular structure with interdependencies randomly distributed. We allow firms in each structure to engage in independent incremental innovation. This yields performance differences across firms and thus allows a sorting among low and high performers. We subsequently allow low-performing firms to imitate highperforming firms. We seek to contrast, all else held constant, the net effect of firm-centered incremental innovation efforts and population-level processes of imitation in the three structures.

The imitation of high performers can take several forms. To avoid favoring one form of imitation over another, we examine several imitation strategies, including: (1) imitation of module decisions, (2) imitation of linkages among decisions, and (3) imitation of both module decisions and linkages. We examine whether these alternative imitation strategies provide asymmetric benefits to innovators and/or imitators.

The first experiment examines perfect imitation. Empirical evidence, however, suggests that imitation of best practices is error-prone even within firms (Szulanski 1996). Therefore, in 
experiment 2 we allow imperfect imitation and contrast three forms: (1) imperfect imitation of module decisions and intra-module dependencies and no imitation of inter-module dependencies, (2) perfect imitation of module decisions and intra-module dependencies but imperfect imitation of inter-module dependencies, and (3) imperfect imitation of module decisions, intra-module dependencies, and inter-module dependencies. This analysis helps address questions about the effective allocation of imitation "energies," either for imitating firms to increase the efficacy of their imitation efforts or for innovating firms to effectively deter imitation.

Our results provide several useful insights on the question of how and why alternative structures affect the trade-off between innovation and imitation deterrence. First, we find that modular structures, in contrast with non-modular ones, generate significant incremental innovation benefits. Further, we find few innovation performance differences between modular and nearly modular structures. More interesting, however, is the finding that the benefits of modular structures are highly susceptible to imitation efforts. In contrast, leader-imitator performance differences persist in the nearly modular and the non-modular structures. These results taken together suggest that if firms expect to be innovators, then nearly modular structures provide the best trade-off between incremental innovation benefits and imitation deterrence. For firms that expect to profit from imitation, modular structures would be their preferred structures, accompanied, of course, by the hope that the innovators also choose modular structures. In contrast, if design complexity is exogenous to firms and designs are modular (e.g., PCs in the case of product design), then innovators cannot rely on design structures to deter imitation efforts.

In the rest of the paper, we describe in detail how we arrived at these conclusions. Section 2 provides a brief background to the literature on imitation, innovation, and complexity. Section 3 outlines the modeling structure that we employed. Section 4 presents the results, and Section 5 concludes. 


\section{Imitation, innovation, and complexity}

Strategy research interest in imitation deterrence grew in lockstep with interest in explaining intra-industry heterogeneity in performance, i.e., why do firms within industries vary in their profitability. Lippman and Rumelt (1982) showed that uncertainty in the choice of production functions was sufficient to generate heterogeneity in profits. The focal point of the theory was the notion of an "isolating mechanism" that prevented the equilibration of industry rents. Rumelt (1984), in pinpointing the isolating mechanism, suggested that "causal ambiguity" - inability to understand the causes of efficiency differences - is the key isolating mechanism.

The notion of causal ambiguity merely pushed the question back one level. What kinds of strategies, under what conditions, are likely to be causally ambiguous? Reed and DeFillippi (1990) suggested that tacitness, complexity, and specificity in a firm's skills and resources generate causal ambiguity that deters imitation efforts. Each of these explanations for causal ambiguity has spawned somewhat independent research efforts. Knowledge-based theories of the firm (Winter 1987; Kogut and Zander 1992; Conner and Prahalad 1996) have sought to explore why tacitness of knowledge can be a barrier to imitation efforts. The transaction cost economics literature has directed attention to the notion of asset specificity and why the presence of specific assets can generate intra-industry heterogeneity (Klein, Crawford, and Alchian 1978; Williamson 1985).

The idea of complexity as the key driver of causal ambiguity, which is the subject matter of this paper, has received much less attention in the strategy literature. Although ideas about complexity have been present in the organization theory literature for several decades (Simon 1957), the exact mechanisms through which complexity deters imitation has a more recent history in strategy. Porter (1991) argued that activity systems form the cornerstone of competitive advantage. "A firm's strategy defines its configuration of activities and how they interrelate" (Porter 1991: 102). The implication is that the multitude of activities that a firm engages in and the interrelationships among them pose a formidable challenge of discovery that, in turn, deters 
imitation. Levinthal (1997), applying the NK fitness landscape structure from Kauffman (1993), shows how clusters of interdependent choices can create persistent heterogeneity in firm strategies. Rivkin (2000), building on the same modeling platform, addressed the question of how and why complexity deters imitation efforts and extends this analysis to consider the dual effect of complexity on firms' own adaptive efforts and other firms' imitation attempts (Rivkin 2001).

In contrast to the strategy literature, which advocates greater complexity as a useful deterrent to imitation, the modularity literature extols the value of modular designs in reducing design complexity. Baldwin and Clark (2000) described how the modular architecture of the System/360 multiplied design options and accelerated design evolution. Although the design benefits of modularity are empirically documented (Garud and Kumaraswamy 1995), our paper is motivated by an often overlooked observation of Baldwin and Clark (2000) that the emergence of modular architectures coincided with the beginning of the decline of IBM's domination in computer hardware. The rise of modular designs contributed to the exploding market for thirdparty plug-compatible devices in the computer industry that competed with IBM's own offerings. This raises the conjecture that IBM, while accelerating design evolution via a modular architecture, perhaps sowed the seeds for the erosion of its competitive advantage by facilitating subsequent imitation efforts.

In a limited attempt at bridging the two views, we see the strategy literature as seeking to direct attention at cognitive complexity, which is a function of overall interdependence among decisions within the firm. ${ }^{1}$ In contrast, the modularity literature directs attention, not at the overall interdependence within the firm, but at the distribution of interdependencies and how they are contained. The intuition is that holding the total number of interdependencies constant but altering their distribution can alter design complexity. This is particularly relevant in the implications for managerial practice. There is little ambiguity about a structure that is more or less modular. It is

\footnotetext{
${ }^{1}$ The idea is that such complexity overwhelms managerial cognitive capacities for strategy making or imitation.
} 
straightforward to visualize and design alternative structures for a given level of interdependencies. In contrast, it is, arguably, harder to implement more or less complex strategies via increasing or decreasing the degree of interdependence within the system since such changes may be constrained by the inherent physics and economics of the underlying system.

The implied contrast between cognitive complexity and design complexity poses an interesting puzzle that provides the motivation for this paper. ${ }^{2}$ On one hand, strategy research seems to indicate that greater cognitive complexity is good from the standpoint of imitation deterrence. On the other hand, research in modularity suggests that reduction in design complexity via modularization can help firms improve flexibility, increase innovation, and engage in speedier response to exogenous environmental change (Baldwin and Clark 2000). This contrast hints at the possibility that while decreases in design complexity have some firm-level benefits, such as greater innovation, they also can have the unintended effect of facilitating easier and quicker imitation. Thus, from the firm's standpoint there may be significant trade-offs to altering design complexity. What form such trade-offs should take is the contribution this paper seeks to advance.

We hold cognitive complexity constant and set up three stylized structures that vary in the level of design complexity. We contrast innovation performance and imitation deterrence across the three structures and in the process shed light on how design complexity facilitates the creation of competitive advantage and its imitation deterrence. The following section describes the model.

\section{Model}

The model set-up for examining the implications of modularization on innovation and imitation requires the specification of three features of the experiment: (1) the representation of the firm and its performance landscape, (2) the characterization of design options, and (3) the representation of innovation and imitation. We elaborate each in turn.

\footnotetext{
${ }^{2}$ Design complexity is closely related to Kolmogorov complexity (Adami 2002), which is a measure of regularity in a system. Kolmogorov complexity will reach its maximum in a disordered system (non-modular system) and its minimum in a well-ordered system (modular system). See Page (1996) for a related distinction.
} 


\subsection{The firm and its performance landscape}

A firm, $f$, is represented as a row vector of $N$ attributes or decision variables, $f=\left\{d_{1} \ldots d_{N}\right\}$. Without loss of generality, each decision can take on one of two possible values $(0,1)$. For instance, a particular decision might be the use of a group-based work organization, with 1 representing the use of groups and 0 representing the absence of groups. However, the performance implications of using group-based work organization cannot be evaluated in isolation. Choice of work organization is likely to have interactions with the incentive system, accounting methods, quality of employees, physical layout, and so on. Thus, some combination of "other" decision choices may yield performance improvements for the focal decision while others may undermine it.

This means that the performance of the firm depends on the setting of the decision variables and the interactions among them. With no interactions between decision variables, each decision makes an independent contribution to overall firm performance. As the interactions between decision variables increase, the contribution of each decision choice to firm performance becomes increasingly interdependent. This means that tweaking a decision choice that results in local performance gains does not always lead to a concomitant increase in firm-level performance. The resulting performance landscape is, therefore, rugged with multiple peaks and valleys, as a result of actions on one decision having ripple effects on other decisions (Levinthal 1997).

The performance contribution $\left(\omega_{i}\right)$ of each decision variable $\left(d_{i}\right)$ is determined both by the state of the $i^{t h}$ decision choice and the states of the $j$ other decision choices on which it depends:

Let $D=\left\{d_{j} \mid j \in\{1, \ldots, N\}\right\}$, then $\omega_{i}=\omega_{i}\left(d_{i} ; D_{i}\right)$, where $D_{i} \subseteq D \forall i \in\{1, \ldots, N\}$

The value of $\omega_{i}$ is treated as an i.i.d. random variable drawn from the uniform distribution $\mathrm{U}[0,1]$ for each $\left(d_{i} ; D_{i}\right)$. Firm performance, $\Omega$, is a simple average of $\omega_{i}$ over the $N$ decisions:

$$
\Omega=\frac{1}{N} \sum_{i=1}^{N} \omega_{i}\left(d_{i} ; D_{i}\right)
$$


If we hold the pattern of interdependencies constant across all firms on a given landscape, it results in $2^{N}$ distinct fitness values, one for each possible configuration of the $N$ decision variables, and corresponds to the canonical $N K$ model from evolutionary biology (Kauffman 1993). Since we are interested in understanding firm performance heterogeneity, we depart from the canonical $N K$ model and allow for heterogeneity in the pattern of interdependencies among firms on a given landscape. Let $R$ be the total number of interdependencies on a given landscape distributed among the $N(N-1)$ cells in an interaction matrix. Thus, allowing for heterogeneity in the distribution of $R$ interdependencies across firms on a given landscape, the total number of distinct performance values is given by, $\left[2^{N}\left(\begin{array}{c}N(N-1) \\ R\end{array}\right)\right]$ where, $R<N(N-1)$. Holding $R$ constant and varying the distribution of the $R$ interdependencies across the $N(N-1)$ cells accomplishes the goal of holding cognitive complexity constant and allowing design complexity to vary.

\subsection{Modeling the design options}

In order to examine the effects of modularity on innovation and imitation, we specify three alternative structures - modular, nearly modular and non-modular - that are identical in $R$, but vary in the degree of modularization.

For each experiment, the number of modules, $M$, and the number of decisions, $N$, are specified. We create $M$ modules, where the $\mathrm{k}^{\text {th }}$ module, $m_{k}$, is comprised of $N / M$ decision variables. We assumed each module to be equal in size to simplify understanding of the results. The composition of each module, $m_{k}$, is determined according to the following rule:

$$
m_{k}=\left\{d_{(k-1) \frac{N}{M}+1}, \ldots ., d_{k \frac{N}{M}}\right\} \text { where, } k \in\{1, \ldots, M\}
$$

We also assign $R$ interdependencies among the pairs of $N$ decisions. In all three structures that we model, $N, M$, and $R$ are held constant. What differs among the three structures is the pattern of distribution of the $R$ interdependencies. 
Differences in the pattern of interdependencies are best understood in terms of an interaction matrix (see Figures 1a-1c). This representation is based on Simon's (1962) exposition of the architecture of complex systems. If $N$ decision variables are present, then the interaction matrix is an $N X N$ array where an $\mathrm{x}$ in cell $c_{i j}$ indicates that the performance contribution of decision $d_{i}$ is dependent on the setting, 0 or 1 , of decision $d_{j}$. In all three designs, we assume that $d_{i}$ depends on $d_{i}$.

\subsubsection{Non-modular structure}

The number of decisions, $N$, number of modules, $M$, and total number of interdependencies, $R$, were specified as above. To generate a non-modular structure, we then created a random number of interactions for each decision, $d_{i}$, subject to the constraint that the total number of interactions for each firm is equal to $R$ (see Figure 1a). This means that, on average, each decision is dependent on $R / N$ other decisions. When looking at Figure 1a, the interaction matrix of the non-modular design setup is an $N X N$ diagonal matrix with $R$ randomly selected offdiagonal elements. In specifying the $R$ interdependencies, each off-diagonal cell had an equally likely chance of being selected.

\subsubsection{Modular structure}

In the modular design setup, we assigned the $R$ interdependencies such that each of the $M$ modules contain $N / M$ decision variables that are tightly coupled, i.e., reciprocally dependent (Thompson 1967). A set of decision variables within a module $m_{k}$ is tightly coupled when the performance value for $d_{i}$ is a function of the $(N / M-1)$ other decision variables in the module. Formally,

$$
\omega_{i}=\omega_{i}\left(d_{i} ; D_{i}\right) \text { where } D_{i}=m_{k} \backslash d_{i} \quad \forall i \in\left\{(k-1) \frac{N}{M}+1, \ldots, k \frac{N}{M}\right\}
$$

In terms of the interaction matrix (Figure 1b), the modular structure corresponds to a blockdiagonal matrix, where each block represents a module. However, what we call modular structures 
do not exactly correspond with Baldwin and Clark's (2000) notion. Their description of modular structures pair block-diagonal structures with visible design rules (see Baldwin and Clark 2000: 74). The pure-form block-diagonal modular structure implemented here merely provides an extreme point of contrast with nearly modular and non-modular structures in our analysis.

\subsubsection{Nearly modular structure}

We made a slight design alteration to the modular set-up to generate the nearly modular structure (see Figure 1c). For each of $(M-1)$ modules, we removed $X$ randomly selected intramodule interdependencies. We re-introduced $X$ interdependencies between randomly selected decisions in the focal module and randomly selected decision variables in the remaining $(M-1)$ modules. This keeps $R$ constant while altering the pattern of interdependencies. Formally, for each module, $m_{k}$, where $k \in\{1, \ldots, M-1\}$, we removed $X$ intra-module linkages resulting in the removal of $X(M-1)$ interdependencies for each firm. For each $X(M-1)$ removals, we uncouple decision $d_{i}$ from decision $d_{j}$, where $i, j \in\left\{(k-1) \frac{N}{M}+1, \ldots, k \frac{N}{M}\right\}$ where $i \neq j$ and $k \in\{1, \ldots, M-1\}$.

To make this structure nearly modular and maintain a constant $R$, we replaced the $X(M-1)$ removed intra-module couplings with $X(M-1)$ inter-module couplings. Formally, we introduced $X(M-1)$ random couplings of decision $d_{h}$ in module $m_{j}$ to decision $d_{i}$ in module $m_{k}$, where, $j \in\{1, \ldots, M-1\}$, and $k \in\{1, \ldots, M\}$ and $j \neq k$ and $h \in\left\{(j-1) \frac{N}{M}+1, \ldots, j \frac{N}{M}\right\}$ and $i \in\left\{(k-1) \frac{N}{M}+1, \ldots, k \frac{N}{M}\right\}$.

As seen in Figure 1c, this generates an interaction for each block below the block-diagonal and removes an interaction for each block within the block-diagonal (with the exception of the last block) when compared to the interaction matrix for the modular design set-up.

The characterization of nearly modular structures raises two important questions: Why are there interactions only below the principal diagonal, and is there is a continuum of nearly modular structures. Our definition of nearly modular structures incorporates two properties - hierarchy and 
near-decomposability - that go back to Simon (1962). The principle of hierarchy imposes a precedence ordering of interdependencies across modules and rules out reciprocal interdependencies between modules. This explains why there are interactions only below the principal diagonal in nearly modular structures. The principle of near-decomposability stipulates that interdependencies within modules should be greater than the interdependencies between modules (see Ethiraj and Levinthal 2004a for a discussion of hierarchy and near-decomposability or loose-coupling). Thus, for a firm with $R$ interdependencies, the structure is nearly decomposable if at least $R / 2$ interdependencies are encapsulated within modules. Thus, we created a continuum of nearly modular structures that meet both criteria. Though we report results only for the case where $X=1$, our results are robust for the full range of nearly modular structures (see online supplement).

\subsection{Modeling Innovation and Imitation}

The main objective of the analysis is to examine how altering design complexity affects the trade-off between innovation and imitation. In each experiment, we allow each firm in the population to engage in incremental innovation attempts. Variance in the success of innovation generates heterogeneity in firm performance. This heterogeneity later fuels imitation efforts. Once firms stabilize on their local peaks, we turn off innovation and allow the low-performing firms to imitate the high-performing firms. ${ }^{3}$ We contrast both innovation performance and imitation deterrence in the three structures. The implementation of innovation and imitation is described below.

\subsubsection{Innovation}

We modeled within-module innovation attempts as a process of incremental local search. Modeled managers attempt to enhance module performance by performing simple intra-module

\footnotetext{
${ }^{3}$ We turn off innovation before allowing imitation because allowing innovation will bias the results in favor of imitators. Once firms stabilize on local peaks, the only way to improve performance is via a "long-jump" (Levinthal 1997). Imitation efforts have the effect of acting like a long-jump and positioning the imitating firms in a new region of the landscape so they can benefit from subsequent local search attempts. For completeness, we also implemented the full set of models in a regime in which innovation and imitation happen in conjunction. All our results in this alternative regime were qualitatively similar to that reported in the paper.
} 
incremental design changes. In each period of the experiment, each module attempts an incremental innovation. Within each module, a randomly selected decision choice is flipped and module performance is evaluated. In this evaluation process, the manager evaluating change assumes there are no changes to other modules. If the change improves performance, then the innovation is implemented. Otherwise, the innovation is discarded (see Ethiraj and Levinthal $2004 \mathrm{~b}$ for a discussion of the behavioral assumptions underlying this form of search).

More formally, for each time period, $t$, and for each module, $m_{k}$, consider a decision choice $d_{j t} \in m_{k}$ that is flipped to $d_{j t}^{\prime}$ (i.e., $0 \rightarrow 1$ or $1 \rightarrow 0$ ). Let module performance be $\Omega_{k t}=\frac{1}{\left|m_{k}\right|} \sum_{i=(k-1) \frac{N}{M}+1}^{k \frac{N}{M}} \omega_{i}\left(d_{i} ; D_{i}\right)$, and let $\Omega_{k t}^{\prime}$ be the module performance with $d_{j t}^{\prime}$ substituted for $d_{j t}$. Then, $d_{j(t+1)}=\left\{\begin{array}{l}d_{j t}^{\prime} \text { if } \Omega_{k t}^{\prime}>\Omega_{k t} \\ d_{j t} \text { otherwise }\end{array}\right.$.

\subsubsection{Imitation}

Imitation is the process by which a low-performing firm replaces a subset of its own decision choices and/or interdependencies with an equivalent set of decision choices and/or interdependencies copied from a high-performing firm. Among the high-performing firms, we assumed that the probability of choosing a particular firm as the target of imitation is proportionate to the firm's performance level (see Goldberg 1989). Implementing imitation involves making at least three behavioral assumptions - unit of imitation (i.e., individual decisions or modules), target of imitation (decisions, linkages, or both), and accuracy of imitation (perfect or imperfect).

We assumed that firms copy clusters of decision choices or linkages rather than individual decision choices or linkages. This assumption has two rationales. First, we believe that managers of imitating firms are generally aware of the dysfunctional effects of imitating individual decisions or linkages when decisions are interdependent. As a result, there are stronger incentives to copy 
clusters of decisions and linkages rather than single decisions or linkages. ${ }^{4}$ Second, imitation is assumed to be rational at the module level in that imitation occurs only if the performance of the target module is superior to the module being considered for replacement. It is also possible to allow imitation based on firm performance improvement. It is far from simple, however, to implement such imitation efforts in reality, because imitation based on firm performance improvement hinges on compensatory evaluation if there are interdependencies across modules. In other words, module managers would have to trade off module performance against firm performance, i.e., accept lower module performance for greater firm performance. Though such compensatory evaluation can result in higher firm performance, it demands the assumption that managers of modules can indeed integrate across module performance and reach the correct decision. This assumption is not supported across a wide swath of field studies and experimental work on evaluation across categories (Dawes 1979; Thaler 1985; Heath and Soll 1996; Read, Loewenstein, and Rabin 1999). This literature suggests that individuals find it cognitively difficult to engage in compensatory evaluation. In addition, implementing compensatory evaluation based on firm performance improvement raises the issue of coordination across modules (and incentives) in imitation decisions. Incorporating these elements is beyond the scope of the research question addressed in this paper.

The issue of targets of imitation turns on the capability to imitate rather than the incentive to imitate. Clearly, firms, if they are able, have an incentive to imitate whole organizations of highperforming firms. Short of imitating whole organizations, however, it is possible to model three alternative imitation targets: (1) module decisions and intra-module linkages, (2) inter-module linkages only, and (3) module decisions, intra-module linkages, and inter-module linkages.

\footnotetext{
${ }^{4}$ Within the model setup, we evaluated five alternative units of imitation: (1) single decisions, (2) single linkages, (3) single decisions and single linkages, (4) modules of decisions, and (5) modules of linkages. We found that imitation of individual decisions (1) or linkages (2) yields no benefits, both in an absolute sense as well as in comparison with alternatives (3), (4), and (5). See Figure OS1 in the online supplement.
} 
There is relatively strong support in the literature that imitating module decisions and linkages is possible and widely observed (Baldwin and Clark 1997; Baldwin and Clark 2000). On the capability question, Heath and Staudenmeyer (2000) suggest that individuals tend to exhibit a strong partition focus (focus on partitioning the task more than on integration) and component focus (focus on single components of a tightly interrelated set of capabilities) respectively. Thus, this experimental work suggests that modules, rather than linkages or individual decisions, would serve as the primary unit of imitation. In reviewing the empirical evidence, Heath and Staudenmeyer (2000) make a compelling case that this tendency is not an agency problem that can be solved with appropriate incentives, but rather a coordination problem rooted in bounded rationality.

The empirical evidence on inter-module linkage copying is sparse. However, experience of firms that implement enterprise software programs such as SAP provides anecdotal evidence of linkage imitation. ERP software comprises standardized modules that are linked via thousands of configuration tables. In the early years of ERP use, there were several highly visible implementation disasters (e.g., Fox-Meyer Drug, Hershey's) that the ERP suppliers learned from and transferred to their later customers (Wah 2000; Anonymous 2002). It appears that much of the changes happened not in modules but in the settings of the configuration tables.

Finally, on the issue of copying both module decisions and linkages, and inter-module linkages, there is little empirical evidence that supports its feasibility or prevalence. Given the strong managerial incentives to do so, however, we implement this extreme form of imitation with the caveat that the interpretation of results should be tempered by the feasibility and capability to do so. In the interest of completeness, we suspend disbelief and leave this judgment to the reader.

With respect to the third assumption about the accuracy of imitation, we varied imitation accuracy to range from perfect to modestly coarse. In cases where imitation is imperfect, we 
assumed that the imitator does not have any information on a subset of choices in the module and makes guesses about them. We describe below the various forms of imitation we modeled.

Module decisions and intra-module linkage copying. Let firm A and firm B denote a highperforming and low-performing firm respectively and the performance of module $k$ in period $t$ in each of the firms is defined as $\Omega_{k t}^{A}$ and $\Omega_{k t}^{B}$. Let module $m_{k}$ be defined as the set of all decision variables $d_{i}$ and their intra-module linkages $\left|D_{i}\right|$. In period $t+1$, Firm B imitates the module decisions and intra-module linkages of firm A according to the following rule:

$m_{k(t+1)}^{B}=\left\{\begin{array}{l}m_{k t}^{A} \text { if } \Omega_{k t}^{A}>\Omega_{k t}^{B} \\ m_{k t}^{B} \text { otherwise }\end{array}\right.$ where, $k \in\{1, \ldots, M\}$ and $m_{k}=\left(d_{i},\left|D_{i}\right|\right)$ where, $i \in\left\{(k-1) \frac{N}{M}+1, \ldots, k \frac{N}{M}\right\}$

Inter-module linkage copying. Let firm A and firm B be a high-performing and low-performing firm respectively and the performance of module $k$ in period $t$ is again defined as $\Omega_{k t}^{A}$ and $\Omega_{k t}^{B}$. Let $\left|D_{j}\right|$ be the set of all inter-module linkages between decisions $d_{i}$ in module $m_{k}$ with all decisions $d_{j}$ in modules $m_{j}$. In period $t+1$, Firm B imitates the inter-module linkages of firm A as $\left|D_{j(t+1)}^{B}\right|=\left\{\begin{array}{l}\left|D_{j t}^{A}\right| \text { if } \Omega_{k t}^{A}>\Omega_{k t}^{B} \\ \left|D_{j t}^{B}\right| \text { otherwise }\end{array}\right.$ where, $k \in\{1, \ldots, M\}$ and $\left|D_{j}\right|=\left(d_{i} ; D_{j}\right)$ where $, i, j \in\left\{(k-1) \frac{N}{M}+1, \ldots, k \frac{N}{M}\right\}$ Module decisions, intra-module linkage copying, and inter-module linkage copying. Let firm A and firm $\mathrm{B}$ be a high-performing and low-performing firm respectively and the performance of module $k$ in period $t$ be defined as $\Omega_{k t}^{A}$ and $\Omega_{k t}^{B}$. Let module $m_{k}$ be defined as the set of all decision variables $d_{i}$, their intra-module linkages by $\left|D_{i}\right|$, and let $\left|D_{j}\right|$ be the set of all inter-module linkages between decisions $d_{i}$ in module $m_{k}$ with decisions $d_{j}$ in modules $m_{j}$. In period $t+1$, Firm B imitates the module decisions, intra-module linkages, and the inter-module linkages of firm A as 
$m_{k(t+1)}^{B}=\left\{\begin{array}{l}m_{k t}^{A} \text { if } \Omega_{k t}^{A}>\Omega_{k t}^{B} \\ m_{k t}^{B} \text { otherwise }\end{array}\right.$ where, $k \in\{1, \ldots, M\}$ and $m_{k}=\left(d_{i},\left|D_{i}\right|\right)$ where, $i \in\left\{(k-1) \frac{N}{M}+1, \ldots, k \frac{N}{M}\right\}$ $\left|D_{j(t+1)}^{B}\right|=\left\{\begin{array}{l}\left|D_{j t}^{A}\right| \text { if } \Omega_{k t}^{A}>\Omega_{k t}^{B} \\ \left|D_{j t}^{B}\right| \text { otherwise }\end{array}\right.$ where, $k \in\{1, \ldots, M\}$ and $\left|D_{j}\right|=\left(d_{i} ; D_{j}\right)$ where $, i, j \in\left\{(k-1) \frac{N}{M}+1, \ldots, k \frac{N}{M}\right\}$

Imperfect imitation. The implementation of imperfect imitation is best understood through an example. Consider firm $A$ attempting to imitate a module from firm $B$. In the perfect copying regime, the module is copied exactly. If copying is imperfect, then a subset of the copied module is unknown. If imitation inaccuracy is 0.1 and there are 5 decisions in the module, then $(0.1 \times 5)$ decision choices will be unknown. Rounding up to the nearest integer, one decision choice is unknown. If the imitation inaccuracy is 0.5 , then three decisions are unknown. A random draw of 0 or 1, representing a guess by the imitating firm, is assigned to each of the unknown decisions. In the limiting case of inaccuracy going to 1 , each decision in the module is assigned a value at random.

More formally, let $G$ be the rate of decision copying inaccuracy, $G \in \mathfrak{R} \mid 0 \leq G \leq 1$. If $G=0$, we have perfect copying accuracy and the decision settings are copied as above. If $G=1$, imitation is equivalent to setting the decisions randomly. For any intermediate value of $G$, let $u$ represent the number of decisions in $m_{k}^{B}$ that is unknown and therefore must be guessed by the imitating firm, where $u \sim B\left(\left|m_{k}^{B}\right|, G\right)$ and $B\left(\left|m_{k}^{B}\right|, G\right)$ is a binomial distribution with parameters $\left|m_{k}^{B}\right|$ and G. Then let $D_{k}=\left\{d_{k}^{1}, \ldots, d_{k}^{u}\right\}$ represent the set of decisions that are unknown and let $D_{k}^{\prime}=\left\{d_{k}^{1^{\prime}}, \ldots, d_{k}^{u^{\prime}}\right\}$ represent the set of guesses made by the imitator, where guess $d_{k}^{i^{\prime}}$ is randomly assigned 0 or 1 with equal probability. Then imperfect imitation of the leader by the imitator in period $t+1$ is

$$
m_{k(t+1)}^{A}=\left(m_{k t}^{B} \cup D_{k}^{\prime}\right) \backslash D_{k} .
$$


Imperfect linkage copying occurs in an analogous manner. Let $G$ be the rate of linkage copying inaccuracy, $G \in \mathfrak{R} \mid 0 \leq G \leq 1$. Let $\left|D_{i}^{B}\right|$ be the set of all intra-module dependencies and $\left|D_{j}^{B}\right|$ the set of all inter-module dependencies for decision $d_{i}$ in module $m_{k}$ in firm B. For any intermediate value of $G$, let $v$ represent the number of decisions in $m_{k}^{B}$ for which the linkages are unknown, and must be guessed by the imitating firm, where $v \sim B\left(\left|m_{k}^{B}\right|, G\right)$ and $B\left(\left|m_{k}^{B}\right|, G\right)$ is a binomial distribution with parameters $\left|m_{k}^{B}\right|$ and $G$. Then let $D_{k}^{B v}=\left\{d_{k}^{1}, \ldots, d_{k}^{v}\right\}$ represent the set of decisions for which their intra-module $\left|D_{i}^{v}\right|$ and inter-module $\left|D_{j}^{v}\right|$ linkages respectively are unknown. For the set of $v$ decisions, the imitating firms retain the status quo of linkages. Thus, imperfect imitation of linkages in period $\mathrm{t}+1$ is defined as

$$
\begin{aligned}
& D_{i(t+1)}^{A}=\left(D_{i t}^{B} \cup D_{i t}^{A v}\right) \backslash D_{i t}^{B v} \text { where } A, B \in\{1, \ldots, F\} \text { and } i \in\left\{(k-1) \frac{N}{M}, \ldots, k \frac{N}{M}\right\} \text { and, } \\
& D_{j(t+1)}^{A}=\left(D_{j t}^{B} \cup D_{j t}^{A v}\right) \backslash D_{j t}^{B v} \text { where } A, B \in\{1, \ldots, F\} \text { and } j \in\left\{(k-1) \frac{N}{M}, \ldots, k \frac{N}{M}\right\} .
\end{aligned}
$$

\section{Analysis}

We performed two experiments. The first experiment contrasts the innovation benefits and imitation deterrence of the three design structures across the three different assumptions about imitation. Experiment 2 examines the robustness of the results in experiment 1 to introducing imperfections in imitation accuracy.

All experiments model the interaction of 100 firms on a given landscape. The initial settings for the decision vectors and interactions are independently specified by random assignment. Since any single run is sensitive to this inherent randomness, we replicate each experiment 100 times with different starting seeds for both the initial state of decision variables and for the interaction matrices. Finally, the term "leaders" refers to the top $10 \%$ of firms in the 
population in each period, and the term "imitators" refers to the bottom $10 \%$ of firms in the population in each period.

\subsection{Experiment 1: Extent of modularity and imitation}

In all the results reported in experiment 1 , we set $N=24, M=4, R=120$, and $G=0$. Holding $R=120$, we varied the spatial distribution of interdependencies to create the three structures shown in Figures 1a-1c. We allowed each firm to engage in $M$ autonomous and parallel incremental innovation attempts in each period of the experiment (i.e., one attempt per module). We implement this hill climbing process until period 100, at which point most firms reach a stable asymptote in performance (i.e., reach a local peak). ${ }^{5}$ After period 100, we turn off innovation, sort the firms, and determine the high and low performers. We then allowed the low performers (henceforth termed imitators) to engage in imitation of the high performers (henceforth termed leaders). In each subsequent period of the experiment, we allowed the imitators to imitate a module from a randomly chosen leader where the leader is chosen with a probability proportionate to population performance. Once an imitation period is complete, we again sort all 100 firms according to their performance and identify the leaders and imitators. As a result, the population of imitators and leaders can change every period as the imitators improve their performance.

Figure 2 graphs the performance levels in the three structures as a function of incremental innovation attempts in the first 100 periods. Comparing the three structures, the non-modular design performs significantly worse than either the modular or nearly modular design. In the nonmodular structure, the partitions for the $M$ modules are drawn with no consideration for grouping highly interdependent decisions. As a result, when one module engages in a local innovation attempt without considering shared interdependencies with other modules, the performance consequences for the firm as a whole are dysfunctional. Innovation attempts that appear to be

\footnotetext{
${ }^{5}$ Firms in the non-modular structure do not reach a stable asymptote by period 100 due to perturbations caused by the off-diagonal interdependencies.
} 
locally performance enhancing frequently turn out to be globally performance decreasing. Thus, the performance of any single firm with a non-modular structure is highly non-monotonic over the 100 periods of the simulation, even though the average over 100 firms, shown in Figure 2, trends upward smoothly (see Figure OS2 in the online supplement for a plot of individual runs).

In contrast, the modular and nearly modular structures exhibit fairly monotonic performance increases as a function of local innovation attempts. In the modular structures, the monotonic increase in performance is because there are no interdependencies between modules. Interestingly, modular and nearly modular structures exhibit nearly identical performance. This is because the ignored interdependencies between modules in the latter case are too few to make a significant difference in the aggregate, and, more subtly, slight deviations from modularity may have a useful consequence by pushing the firm off inferior local peaks. The one-step incremental innovation process localizes its impact within modules and only rarely do innovation attempts within a module affect the performance of another module. Thus, from the standpoint of incremental innovation, it appears that the efficacy of modular and nearly modular structures is nearly identical. ${ }^{6}$

\subsubsection{Imitation of module decisions and intra-module linkages}

Figure 3 graphs the results of the experiment comparing the three structures over the entire experiment. For ease of comparison, we plot the average difference in the performance of the leaders and imitators in the three structures at every period of the experiment. In the non-modular structure, the average leader-imitator performance difference reaches about 0.20 by period 100 when we turn off innovation. In the modular and nearly modular structures, the average leaderimitator performance difference is about 0.10 and 0.11 respectively.

\footnotetext{
${ }^{6}$ Note that our results comparing innovation performance across the three structures is strictly confined to incremental innovation only. It is possible that modular and nearly modular structures may actually hamper radical, system-wide innovation efforts (see Ulrich 1995).
} 
The examination of Figure 3 from period 101 onwards helps identify the imitation deterrence potential across the three structures. In the non-modular structure, the average leaderimitator performance difference increases sharply after imitation is implemented, and this difference remains stable until termination of the simulation in period 500. Interestingly, this increase in leader-imitator performance difference is not driven by an increase in the performance of the leaders, which remains constant throughout. The change is driven by the decrease in performance of imitators as a result of dysfunctional effects of imitation. Imitation in non-modular structures destroys some of the value of the prior successful innovation attempts of the imitators. The intuition underlying these results is rooted in the interdependencies between modules. Recall from the description of the model, the linkages between modules are heterogeneous across firms. As a result, when imitators copy modules from leaders, they copy only the module decisions and the dependencies within modules. Since the distribution of inter-module dependencies of leaders is different from the distribution of inter-module dependencies of imitators, imitation efforts hurt the performance of imitators and thus increase the average leader-imitator performance difference. ${ }^{7}$

In the modular and nearly modular structures, the average leader-imitator performance difference declines from period 101 onwards. Whereas in the modular structure the difference completely disappears by period 325 , in the nearly modular case the performance difference persists and remains stable. The intuition behind this result deserves elaboration. In the modular structure, all firms in the population share the same groupings of decisions. Thus, performance differences are a function of differences in the decision settings. Since there are no inter-module interdependencies, imitation of module decisions and intra-module linkages from high-performing firms results in the gradual convergence of the population on the same decision choices. In the

\footnotetext{
${ }^{7}$ Consider, for example, Kmart attempting to imitate Wal*mart's logistics system. Kmart may have made more or less the same investments in individual modules such as a trucking fleet, cross-docking system, satellite-guided fulfillment, and communication networks with suppliers. The main difference in realized performance might lie not in the modules but in how one module utilizes information from another, i.e., in the linkages between modules. These linkages of information sharing, coordination mechanisms, and allocation of decision rights within the organization may remain idiosyncratic even in the presence of module imitation.
} 
nearly modular structure, however, firms differ in the distribution of interdependencies between modules. Since imitating firms copy only modules and do not copy the linkages between modules, they are unable to mimic the performance of the leaders. Intuitively, the difference between modular and nearly modular structures in their imitation deterrence lies in the process of imitation. Imitation, unlike innovation, proceeds in larger increments (i.e., modules). Thus, each imitation attempt affects other modules within the firm and their disruptive effect is amplified, particularly if the interdependencies between modules are heterogeneous across firms. The effect of even small interdependencies between modules that are ignored in imitation multiplies over time and hinders the complete imitation of high-performing firms. This suggests that the average leader-imitator performance difference should be increasing in the extent of inter-module dependencies which we confirmed in experiments not reported here. ${ }^{8}$ Thus, inter-module linkages have a strong imitation deterring property if we assume that imitators copy only module decisions and intra-module linkages.

\subsubsection{Imitation of module decisions, intra-module linkages, and inter-module linkages ${ }^{9}$}

In the experiment above, with imitation of modules (decisions and intra-module linkages), we saw that the leader-imitator performance differences were driven primarily by heterogeneity in inter-module linkages and firms' inability to imitate such linkages. We retained the assumption that inter-module linkages are heterogeneous but relaxed the assumption that they cannot be imitated. All other settings in the model were identical to that described in Section 4.1.1.

Figure 4 presents the results of model runs with perfect imitation of both decisions and linkages (intra-module and inter-module). Contrasting Figure 4 with Figure 3 reveals the implication of assuming that firms can (or cannot) imitate module linkages. In the modular

\footnotetext{
${ }^{8}$ See Figure OS3 in the online supplement.

${ }^{9}$ We also ran a set of models where only inter-module linkages are copied. These results were qualitatively similar to the results where only module decisions and intra-module linkages are copied. See Figure OS4 in the online supplement.
} 
structure, this assumption has no impact since there are no inter-module dependencies. In the nearly modular structure, however, the leader-imitator performance difference goes down to almost zero. The difference does not completely disappear because we implement imitation based on module performance rather than firm performance. An imitating firm might refrain from copying a module because her existing module has higher performance. However, at the firm-level the modest interdependencies between modules reduce the performance of the imitating firm in comparison with the leader. If we implement imitation based on firm performance, then the leaderimitation performance difference will go down to zero. This finding confirms the intuition that a key lever observed in Figure 3 is the inability to imitate inter-module linkages.

Apart from the contrast with Figure 3, the striking finding in Figure 4 is that the average leader-imitator performance differences continue to persist in the non-modular structures and to a marginal extent in the nearly modular structure as well. This runs counter to our expectations that imitators endowed with the perfect ability to imitate modules and inter-module linkages should over time be able to mimic the performance of the leaders. The starting point for the intuition here is again the heterogeneity in inter-module linkages. Recall from Section 3.3.2 that we implement intelligent imitation of modules, wherein the imitator copies a module and its inter-module linkages from a leader if the performance of the target module is greater than the performance of its own module. Much of the persistent performance difference arises from one (or more) module(s) that the imitators do not copy from leaders. This happens because the performance of the corresponding module within the imitator firm is higher than that of the leader firm. However, at the firm level the interdependence between the higher-performing retained module (in imitators) and the other imitated modules produces lower performance as compared with the leaders.

Consider a simple illustration. Firm A is a leader with average module performances being $(0.74,0.78,0.82$, and 0.85$)$. Firm B is the imitator with average module performances being $(0.78$, $0.75,0.82$, and 0.81 ). Firm B has perfectly copied modules 2,3 , and 4 and their linkages from Firm 
A, but has not copied module 1 since it has a higher performance than the corresponding module in Firm A. However, module 1 in Firm B shares interdependencies with modules 2 and 4 such that the higher performance of module 1 in Firm A as compared with Firm B is more than offset by the poorer performance of modules 2 and 4 . In this case, there is a persistent performance difference of about 0.03 . Thus, idiosyncratic inter-module linkages coupled with intelligent imitation causes persistent leader-imitator performance differences even when imitators are endowed with the ability to copy modules and their linkages perfectly. Once again, the leader-imitator performance difference is increasing in the level of inter-module dependencies and reaches its maximum in nonmodular structures (see Figure OS5 in the online supplement for additional analyses). It is important to note that two important assumptions drive this result: heterogeneity in inter-module linkages and intelligent imitation. For instance, if we replaced intelligent imitation with the random, but perfect, imitation of the individual decisions and linkages, then the leader-imitator performance difference will eventually go down to zero in all three structures. ${ }^{10}$ Thus, evaluating the insight from our model results turns on the question of whether the twin assumptions of heterogeneity in inter-module linkages and intelligent imitation of modules are in fact behaviorally plausible assumptions.

The result that the extent of modularity creates a trade-off between maximizing innovation and deterring imitation is distinct from, but broadly similar in spirit to, Rivkin's (2001) findings that firms with moderately complex strategies provide the best trade-off between facilitating replication (imitation by the focal firm) and deterring imitation. In our analysis, in contrast to Rivkin (2001), we hold constant the number of interdependencies and vary the spatial distribution of interdependencies to generate structures that vary in the extent of modularity. Thus, while Rivkin (2001) finds that greater or lesser interdependence is pertinent to the trade-off between replication and imitation, our results suggest that even holding interdependence constant, varying

\footnotetext{
${ }^{10}$ See Figure OS6 in the online supplement for a model of random module imitation based on firm performance gains.
} 
design complexity (i.e., ranging from modular to non-modular) affects the trade-off between innovation and imitation deterrence.

These results raise the question of competition among alternative structures and how evolutionary selection forces might differentially favor or penalize them. Comparing the average performance and standard deviation over time across the three structures provides some clues. As seen from Figure 5, if selection forces play out in the first 100 periods, then modular and nearly modular structures are likely to be favored since their average performance is higher and standard deviation is lower. The picture reverses if selection occurs after 250 periods. By then, the variance among modular structures goes to zero and their average performance is the lowest. Selection at this stage is most likely to favor the nearly modular structures. This provides the rationale for why Baldwin (personal communication) argues that modularity presents implications that are “compelling, surprising, and dangerous." If in the early stages of an industry firms are faced with a choice of alternative structures and selection is expected to unfold rapidly, then choosing modular structures seems "compelling." However, the "dangerous" implication is that the imitation deterrence potential of this choice is limited and competition in the later stages of industry evolution is less likely to be based on innovation. The "surprising" element is seen if selection pressures are delayed and a variety of structures are allowed to coexist for a significant period of time. In such a circumstance, modular structures are likely to be selected out. Thus, in the face of this conundrum, nearly modular structures appear to be the least risky choice for managers since they balance both short-term and long-term considerations.

\subsection{Experiment 2: Imperfect imitation}

In experiment 1 , while we varied the basis of imitation, we assumed that imitation was achieved with perfect accuracy. This assumption is at odds with much empirical evidence (Garud and Nayyar 1994; Szulanski 1996; Simonin 1999). In experiment 2, we relaxed this assumption and varied imitation accuracy, otherwise retaining all the model settings in experiment 1 . In all 
models, we set $G=0.10$. We report two model runs. The first assumes that imitators imperfectly imitate module decisions and intra-module linkages and do not imitate inter-module linkages in order to facilitate a comparison with Figure 3. The second assumes that imitators perfectly imitate module decisions and intra-module linkages, but imperfectly imitate module linkages. ${ }^{11}$ Assuming that leaders (imitators) are budget constrained and have to expend resources to improve imitation deterrence (accuracy), this exercise will afford a better understanding of what their relative allocation of budgets should emphasize - the modules (decisions and linkages) or the inter-module linkages.

Figure 6 presents the results of model runs with imperfect imitation of module decisions and intra-module linkages. The figure shows that with a reduction in imitation accuracy, the salience of design complexity is reduced. On average, inaccurate copying of even one decision in each module of modular structures is sufficient to improve their imitation deterrence potential. Note, however, that in the case of modular structures, performance differences are completely driven by inaccuracies in decision imitation since all decisions in a module are tightly coupled, thus making intra-module linkage copying redundant. In this regime, imitators would be better off not imitating at all. On the flip side, leaders can afford to pay less attention to design complexity from the standpoint of imitation deterrence if they can hamper imitation accuracy. ${ }^{12}$

In the nearly modular and non-modular structures, however, the differences continue to persist since intra-module linkages are sparse and are thus copied inaccurately. Linkage copying affects the results via two mechanisms. First, the parameter $G$ drives the number of linkages that are unknown and thus should be guessed. Second, the accuracy of guesses is a function of the

\footnotetext{
${ }^{11}$ We also engaged in two other sets of models: (1) only inter-module linkages are copied imperfectly, and (2) module decisions, intra-module linkages, and inter-module linkages are all copied imperfectly. See Figures OS7 and OS8 in the online supplement.

${ }^{12}$ This raises the question whether imitators can discover and correct imitation efforts. This is a function of the number of decisions that are copied inaccurately. If only a single decision is copied inaccurately then it is possible to recover via subsequent local search. As the number of imperfectly copied decisions increases, subsequent local search will not eliminate leader-imitator performance differences.
} 
space of interactions. The larger the space of possible interactions over which the guesses can be made, the greater the inaccuracy of imitation. ${ }^{13}$

This then begs the question as to whether imitators also can discover via a local search process the intra-module linkages. In general, it is accepted that the discovery of linkages among decisions is an NP-hard problem (Schaefer 1999; Ethiraj and Levinthal 2004a; Aragones et al. 2005). As intra-module linkages (see footnote 13) become sparse, the number of alternative configurations that imitators would need to explore will skyrocket and thus render impractical any efforts at independently discovering the linkages. Thus, consistent with this prior research, we believe that inaccuracies in copying intra-module linkages are sufficient to preserve leader-imitator performance differences in nearly modular and non-modular structures.

Figure 7 presents the results of models with perfect imitation of module decisions and intramodule linkages but imperfect imitation of inter-module linkages. In the modular structure, the average leader-imitator performance difference again goes to zero since there are no inter-module linkages to imitate and imitators perfectly copy module decisions. This suggests the corollary that average leader-imitator performance differences should be increasing in the level of inter-module linkages. The results for the nearly modular and non-modular structures confirm this intuition.

This again begs the question as to whether imitators can discover and correct the inaccurate inter-module linkages via an experimentation process. The answer here is not identical to that above. Prior research suggests that the ease with which firms can discover linkages depends on whether linkages are reciprocal or sequential. Ethiraj and Levinthal (2004a) show that the problem of discovering sequential (or one-way) linkages is solvable via a simple local search process without exhaustive enumeration. Reciprocal linkages between decisions, however, severely erode the efficacy of local search, and discovering linkages is not possible without exhaustive

\footnotetext{
${ }^{13}$ More precisely, if the space of interactions is ' $n$ ' and the number of guesses is ' $r$ ', then the number of possible combinations equals $\frac{n !}{r !(n-r) !}$. Thus, imitation inaccuracy will be highest when $\mathrm{n}=\mathrm{r}$.
} 
enumeration. This means that in nearly modular structures, it may be possible for imitators to discover inaccurate inter-module linkages via a local search process and thus bridge some of the leader-imitator performance differences because the nearly modular structures incorporate only sequential linkages between modules. In contrast, in the non-modular structure, imitators are unlikely to be able to independently discover inter-module linkages since they are reciprocal.

In sum, the results of experiment 2 enrich the results from experiment 1 . First, modest inaccuracies in imitating decisions are sufficient to generate imitation deterrence even for leaders with modular structures. Second, imperfect imitation of linkages substantially amplifies the imitation deterrence effect of nearly modular structures. In all cases, the relative ranking of the alternative designs is the same for perfect and imperfect imitation of decisions and linkages.

\section{Discussion}

The architecture of complex systems (Simon, 1962) clearly has important implications for how firms adapt. However, the focus has been on how firms may increase their performance and respond to a changing environment. The dual implications of different structures on adaptive and imitative processes have remained unexplored. This gap is particularly salient in recent discussions of the power of modular systems. Using a simple model, we show that nearly modular structures tend to provide greater incremental innovation benefits as compared with non-modular structures and better imitation deterrence as compared to fully modular structures. The interesting question from a managerial standpoint is what does it mean to alter design complexity or the pattern of interdependencies between departments of an organization or between product components that in a real way affects the performance of firms while simultaneously deterring imitation?

Altering the interdependencies between departments of an organization or components of products has real performance implications as well as imitation-deterrence potential. Take the example of the airline pricing wars of the 1980s or the introduction of frequent flier programs. Both pricing strategies and the introduction of frequent flier programs were fairly modular in 
nature. Global price changes in flights were highly visible and public and had little relationship with other activities of the airlines such as flight paths, schedules, maintenance activities, or staffing. Imitation of pricing strategy simply involved making changes in fare schedules. Thus, there was a swift retaliatory imitation of price cuts by all airlines. The introduction of frequent flier programs also followed a similar imitation pattern. In contrast, Southwest Airlines developed a partially coupled set of mutually reinforcing strategies such as low cost, short-distance point-topoint flights, quick turnaround of planes, and low maintenance costs from the use of standardized aircraft. Imitation of parts of the strategy by other airlines did not generate the same payoffs simply because it ignored the interdependencies with other parts. In addition, the tightly coupled or nonmodular nature of the strategies pursued by larger carriers, such as Delta, impeded effective local adaptation while disrupting their partial imitation attempts (Rivkin and Therivel 2004).

Thus, in our view, nearly modular structures outperform the non-modular or modular structures because they support a significant degree of localized adaptation while simultaneously retaining sufficient interdependence with other mutually reinforcing activities such that partial imitation generally does not yield the same benefits. In other words, the performance gains from complete modularization come at the expense of ignoring the long-term durability of such gains. From the standpoint of managerial practice, our notion of increasing or decreasing design complexity amounts to altering the organization of interdependencies within a firm, rather than increasing or decreasing total interdependence. The overall level of interdependence may be heavily constrained by the inherent nature of physical systems (Baldwin and Clark 2000) or the underlying economics of a business system. However, how the interdependence is managed and organized is clearly an instrument of design. The main contribution of our study is in explicating the trade-offs inherent in real, observable design choices that firms make.

It is useful to also consider the normative implications that emerge from our analysis, particularly the question of what structure managers should choose if they are concerned with 
managing the trade-off between innovation and imitation deterrence. This is clearly a difficult question to answer without a full picture of the setup costs associated with the three structures. Much like the theory of the firm debate (i.e., whether firms are superior to markets), the question turns on the issue of whether the default state is fully modular or non-modular. On the one hand, if we assume that in the beginning there were non-modular structures and firms need to expend resources to attain modular structures, then firms will be better off not getting to the extreme world of modular designs. Simply encapsulating reciprocal dependencies within modules and limiting all inter-module dependencies to be sequential will suffice to provide an effective trade-off between innovation and imitation deterrence. On the other hand, if we assume that modular structures are the default and firms need to expend resources to make them less modular, then the implications are less sanguine. If selection is expected to operate in the early phases of industry evolution, then expending resources to move toward less modular structures will create not only a cost disadvantage but also a short-term disadvantage in facing selection pressures and poses a real threat of eliminating less modular structures, albeit prematurely. Overall, though, nearly modular structures appear to provide a reasonable insurance both against short-term and long-term competitive pressures.

Third, if choices about design complexity are beyond the control of individual firms in the industry (e.g., the PC industry), such as when product standards are controlled by standards bodies such as IEEE, then design complexity is unlikely to have significant implications for imitation deterrence. For instance, if systems are modular and this choice is exogenous to firms, then innovators cannot rely on design complexity to deter imitation efforts. Thus, the usefulness of design complexity as an instrument of managerial strategy is contingent on the choices about complexity being endogenous to firms. While this is perhaps possible in the case of organization design choices, it is less apparent in the case of technology products where industry participants often need to agree on architectural specifications and design standards. 
The present analysis, however, remains incomplete in a variety of respects. First, we explore only the trade-off between innovation and imitation deterrence. This captures only a small slice of the full picture of trade-offs that accompany design decisions such as extent of modularity and ignores a variety of other competitive considerations that accompany discussions of modularity such as standards, interfaces, inter-firm supplier relationships, technological domains, industry life cycle and so on. Second, we explore only how design complexity affects incremental innovation and modular imitation. Our analysis does not speak to the issue of how design complexity affects radical or architectural innovation. Arguably, modular designs may significantly hamper radical innovation since it demands coordination across the full system (Ulrich 1995). Third, we assume that the pattern of interdependencies is constant over time. If changes in interdependencies occur exogenously, then the ordering of the three structures with respect to innovation benefits and imitation deterrence may also change.

Nonetheless, the current work extends the literature's prior treatment by jointly considering the adaptive consequences of changing to a more modular structure and the competitive implications that result from the impact of modularity on the diffusion of organizational practices. Baldwin and Clark's (2000) depiction of the radical decline in absolute and relative value of IBM with the adoption of a modular product architecture is a powerful testament to the importance of examining the dual role of modularity in enhancing innovation and facilitating imitation. Design choices do not take place in a competitive vacuum of a firm's own performance along a single measure of performance such as innovation, but in a competitive context with the threat of imitation. A full consideration of design choices must recognize both facets. 


\section{References}

Adami, C. (2002). What is complexity? BioEssays, 24, 1085-94.

Anonymous (2002). 35 years of IT leadership: The best and the worst, in Computerworld Vol. 36.

Aragones, E., I. Gilboa, A. Postlewaite, and D. Schmeidler (2005). Fact-free learning, American Economic Review, 95 (5), 1355-68.

Baldwin, C. Y. and K. B. Clark (1997). Sun wars: Competition within a modular cluster, 19851990, in Competing in the age of digital convergence, D. B. Yoffie, Ed. Boston, MA: Harvard Business School Press.

Baldwin, C. Y. and K. B. Clark (2000). Design rules: The power of modularity. Cambridge, MA: The MIT Press.

Barney, J. (1991). Firm Resources and Sustained Competitive Advantage, Journal of Management, 17 (1), 99-120.

Conner, K. R. and C. K. Prahalad (1996). A resource-based theory of the firm: Knowledge versus opportunism, Organization Science, 7 (5), 477-501.

Dawes, R. M. (1979). Robust Beauty of Improper Linear-Models in Decision-Making, American Psychologist, 34 (7), 571-82.

Ethiraj, S. K. and D. A. Levinthal (2004a). Bounded rationality and the search for organizational architecture: An evolutionary perspective on the design of organizations and their evolvability, Administrative Science Quarterly, 49 (3), 404-37.

Ethiraj, S. K. and D. A. Levinthal (2004b). Modularity and innovation in complex systems, Management Science, 50 (2), 159-73.

Garud, R. and A. Kumaraswamy (1995). Technological and organizational designs for realizing economies of substitution, Strategic Management Journal, 16 (Special Issue), 93-109.

Garud, R. and P. R. Nayyar (1994). Transformative capacity: Continual structuring by intertemporal technology transfer, Strategic Management Journal, 15, 365-86.

Goldberg, D. E. (1989). Genetic algorithms in search, optimization and machine learning. Reading, MA: Addison-Wesley Longman.

Heath, C. and J. B. Soll (1996). Mental accounting and consumer decisions, Journal of Consumer Research, 23, 40-52.

Heath, C. and N. Staudenmayer (2000). Coordination neglect: How lay theories of organizing complicate coordination in organizations, Research in Organizational Behavior, 22, 153-91. 
Kauffman, S. A. (1993). The origins of order: Self-organization and selection in evolution. New York: Oxford University Press.

Klein, B., R. G. Crawford, and A. A. Alchian (1978). Vertical Integration, Appropriable Rents, and the Competitive Contracting Process, Journal of Law \& Economics, 21 (2), 297-326.

Kogut, B. and U. Zander (1992). Knowledge of the firm, combinative capabilities and the replication of technology, Organization Science, 3 (3), 383-97.

Levinthal, D. A. (1997). Adaptation on rugged landscapes, Management Science, 43 (7), 934-50.

Lippman, S. and R. Rumelt (1982). Uncertain imitability: An analysis of interfirm differences in efficiency under competition, Bell Journal of Economics, 13 (2), 418-53.

Page, S. E. (1996). Two measures of difficulty, Economic Theory, 8 (2), 321-46.

Parnas, D. L. (1972). On the criteria to be used in decomposing systems into modules, Communications of the ACM, 15 (12), 1053-58.

Porter, M. E. (1980). Competitive strategy: techniques for analyzing industries and competitors. New York: Free Press.

Porter, M. E. (1991). Towards a Dynamic Theory of Strategy, Strategic Management Journal, 12 (Winter), 95-117.

Read, D., G. Loewenstein, and M. Rabin (1999). Choice bracketing, Journal of Risk \& Uncertainty, 19 (1-3), 171-97.

Reed, R. and R. J. DeFillippi (1990). Causal ambiguity, barriers to imitation, and sustainable competitive advantage, Academy of Management Review, 15 (1), 88-102.

Rivkin, J. (2000). Imitation of complex strategies, Management Science, 46 (6), 824-44.

Rivkin, J. (2001). Reproducing knowledge: Replication without imitation at moderate complexity, Organization Science, 12 (3), 274-93.

Rivkin, J. and L. Therivel (2004). Delta Air Lines (A): The low-cost carrier threat. Boston, MA: Harvard Business School Case.

Rumelt, R. P. (1984). Toward a strategic theory of the firm, in Competitive Strategic Management, R. B. Lamb, Ed. Englewood Cliffs: NJ: Prentice-Hall.

Schaefer, S. (1999). Product design partitions with complementary components, Journal of Economic Behavior \& Organization, 38 (3), 311-30. 
Simon, H. A. (1957). Administrative behavior: A study of decision-making processes in administrative organization (2nd. ed.). New York: Macmillan.

Simon, H. A. (1962). The architecture of complexity, Proceedings of the American Philosophical Society, 106, 467-82.

Simonin, B. L. (1999). Ambiguity and the process of knowledge transfer in strategic alliances, Strategic Management Journal, 20 (7), 595-623.

Szulanski, G. (1996). Exploring internal stickiness: Impediments to the transfer of best practice within the firm, Strategic Management Journal, 17 (Winter), 27-43.

Thaler, R. H. (1985). Mental accounting and consumer choice, Marketing Science, 4, 199-214.

Thompson, J. D. (1967). Organizations in action. New York: McGraw-Hill.

Ulrich, K. T. (1995). The role of product architecture in the manufacturing firm, Research Policy, $24,419-40$.

Wah, L. (2000). Give ERP a chance, Management Review, 89 (3), 20-24.

Williamson, O. E. (1985). Economic Institutions of Capitalism: Firms, Markets, Relational Contracting. New York: The Free Press.

Winter, S. G. (1987). Knowledge and competence as strategic assets, in The Competitive Challenge, D. Teece, Ed. Cambridge, MA: Ballinger.

Winter, S. G. (1995). The four Rs of profitability: Rents, resources, routines, and replication, in Resource-based and evolutionary theories of the firm: Towards a synthesis, C. A. Montgomery, Ed. Boston, MA: Kluwer Academic Publishers. 
Figure 1a. Non-modular Structure

\begin{tabular}{|c|c|c|c|c|c|c|c|c|c|c|c|c|}
\hline & 1 & 2 & 3 & 4 & 5 & 6 & 7 & 8 & 9 & 10 & 11 & 12 \\
\hline 1 & $\mathbf{x}$ & & & $\mathbf{X}$ & $\mathbf{X}$ & & & & & $\mathbf{x}$ & & \\
\hline 2 & $\mathbf{X}$ & $\mathbf{X}$ & $\mathbf{X}$ & & & $\mathbf{X}$ & $\mathbf{X}$ & $\mathbf{X}$ & & & & $\mathbf{x}$ \\
\hline 3 & & & $\mathbf{x}$ & & & $\mathbf{X}$ & $\mathbf{X}$ & $\mathbf{X}$ & & & & $\mathbf{x}$ \\
\hline 4 & & $\mathbf{X}$ & & $\mathbf{X}$ & & & $\mathbf{X}$ & & & & & \\
\hline 5 & & & & $\mathbf{X}$ & $\mathbf{X}$ & & & & & & & $\mathbf{X}$ \\
\hline 6 & $\mathbf{X}$ & & & & $\mathbf{x}$ & $\mathbf{X}$ & & & & & & $\mathbf{X}$ \\
\hline 7 & & & $\mathbf{X}$ & & & & $\mathbf{X}$ & & $\mathbf{x}$ & $\mathbf{X}$ & & $\mathbf{x}$ \\
\hline 8 & & & & & 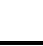 & & $\mathbf{x}$ & $\mathbf{x}$ & & & $\mathbf{x}$ & $\mathbf{x}$ \\
\hline 9 & & & & & & & & $\mathbf{X}$ & $\mathbf{X}$ & & & \\
\hline 10 & & & & $\mathbf{X}$ & $\mathbf{X}$ & & & & $\mathbf{x}$ & $\mathbf{x}$ & & \\
\hline 11 & & $\mathbf{X}$ & & & $\mathbf{x}$ & & & $\mathbf{X}$ & $\mathbf{x}$ & & $\mathbf{x}$ & \\
\hline 12 & & & & & & & & & & & $\mathbf{x}$ & $\mathbf{x}$ \\
\hline
\end{tabular}

Figure 1b. Modular Structure

\begin{tabular}{|c|c|c|c|c|c|c|c|c|c|c|c|c|}
\hline & 1 & 2 & 3 & 4 & 5 & 6 & 7 & 8 & 9 & 10 & 11 & 12 \\
\hline 1 & $\mathbf{X}$ & $\mathbf{X}$ & $\mathbf{X}$ & $\mathbf{X}$ & & & & & & & & \\
\hline 2 & $\mathbf{X}$ & $\mathbf{X}$ & $\mathbf{x}$ & $\mathbf{X}$ & & & & & & & & \\
\hline 3 & $\mathbf{X}$ & $\mathbf{x}$ & $\mathbf{x}$ & $\mathbf{x}$ & & & & & & & & \\
\hline 4 & $\mathbf{X}$ & $\mathbf{x}$ & $\mathbf{x}$ & $\mathbf{x}$ & & & & & & & & \\
\hline 5 & & & & & $\mathbf{x}$ & $\mathbf{X}$ & $\mathbf{X}$ & $\mathbf{X}$ & & & & \\
\hline 6 & & & & & $\mathbf{X}$ & $\mathbf{x}$ & $\mathbf{X}$ & $\mathbf{x}$ & & & & \\
\hline 7 & & & & & $\mathbf{X}$ & $\mathbf{X}$ & $\mathbf{x}$ & $\mathbf{x}$ & & & & \\
\hline 8 & & & & & $\mathbf{X}$ & $\mathbf{x}$ & $\mathbf{x}$ & $\mathbf{x}$ & & & & \\
\hline 9 & & & & & & & & & $\mathbf{X}$ & $\mathbf{X}$ & $\mathbf{X}$ & $\mathbf{X}$ \\
\hline 10 & & & & & & & & & $\mathbf{x}$ & $\mathbf{x}$ & $\mathbf{x}$ & $\mathbf{x}$ \\
\hline 11 & & & & & & & & & $\mathbf{x}$ & $\mathbf{x}$ & $\mathbf{x}$ & $\mathbf{x}$ \\
\hline 12 & & & & & & & & & $\mathbf{x}$ & $\mathbf{x}$ & $\mathbf{x}$ & $\mathbf{x}$ \\
\hline
\end{tabular}

Figure 1c. Nearly modular Structure

\begin{tabular}{|c|c|c|c|c|c|c|c|c|c|c|c|c|}
\hline & 1 & 2 & 3 & 4 & 5 & 6 & 7 & 8 & 9 & 10 & 11 & 12 \\
\hline 1 & $\mathbf{X}$ & $\mathbf{X}$ & $\mathbf{X}$ & $\mathbf{X}$ & & & & & & & & \\
\hline 2 & $\mathbf{x}$ & $\mathbf{X}$ & & $\mathbf{X}$ & & & & & & & & \\
\hline 3 & $\mathbf{X}$ & $\mathbf{X}$ & $\mathbf{X}$ & $\mathbf{X}$ & & & & & & & & \\
\hline 4 & $\mathbf{x}$ & $\mathbf{X}$ & $\mathbf{X}$ & $\mathbf{X}$ & & & & & & & & \\
\hline 5 & & & & & $\mathbf{X}$ & $\mathbf{X}$ & $\mathbf{x}$ & $\mathbf{X}$ & & & & \\
\hline 6 & & $\mathbf{X}$ & & & $\mathbf{x}$ & $\mathbf{x}$ & $\mathbf{x}$ & $\mathbf{X}$ & & & & \\
\hline 7 & & & & & & $\mathbf{X}$ & $\mathbf{x}$ & $\mathbf{X}$ & & & & \\
\hline 8 & & & & & $\mathbf{x}$ & $\mathbf{x}$ & $\mathbf{x}$ & $\mathbf{x}$ & & & & \\
\hline 9 & & & & & & & & & $\mathbf{X}$ & $\mathbf{X}$ & $\mathbf{X}$ & $\mathbf{X}$ \\
\hline 10 & & & & & & & & & $\mathbf{x}$ & $\mathbf{x}$ & $\mathbf{x}$ & $\mathbf{x}$ \\
\hline 11 & & & & & & & $\mathbf{X}$ & & $\mathbf{x}$ & $\mathbf{X}$ & $\mathbf{x}$ & $\mathbf{x}$ \\
\hline 12 & & & & & & & & & $\mathbf{x}$ & $\mathbf{x}$ & $\mathbf{x}$ & $\mathbf{x}$ \\
\hline
\end{tabular}



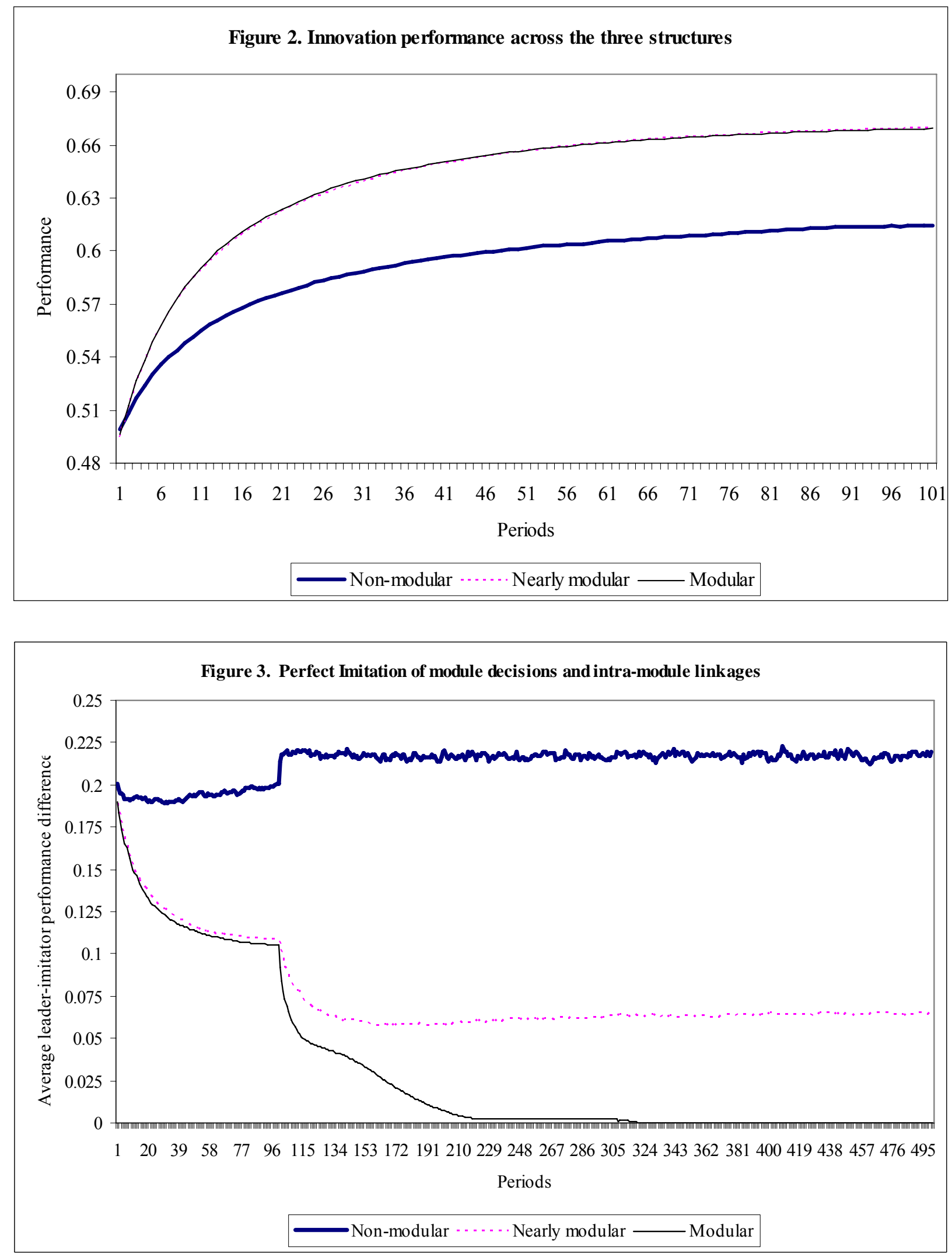

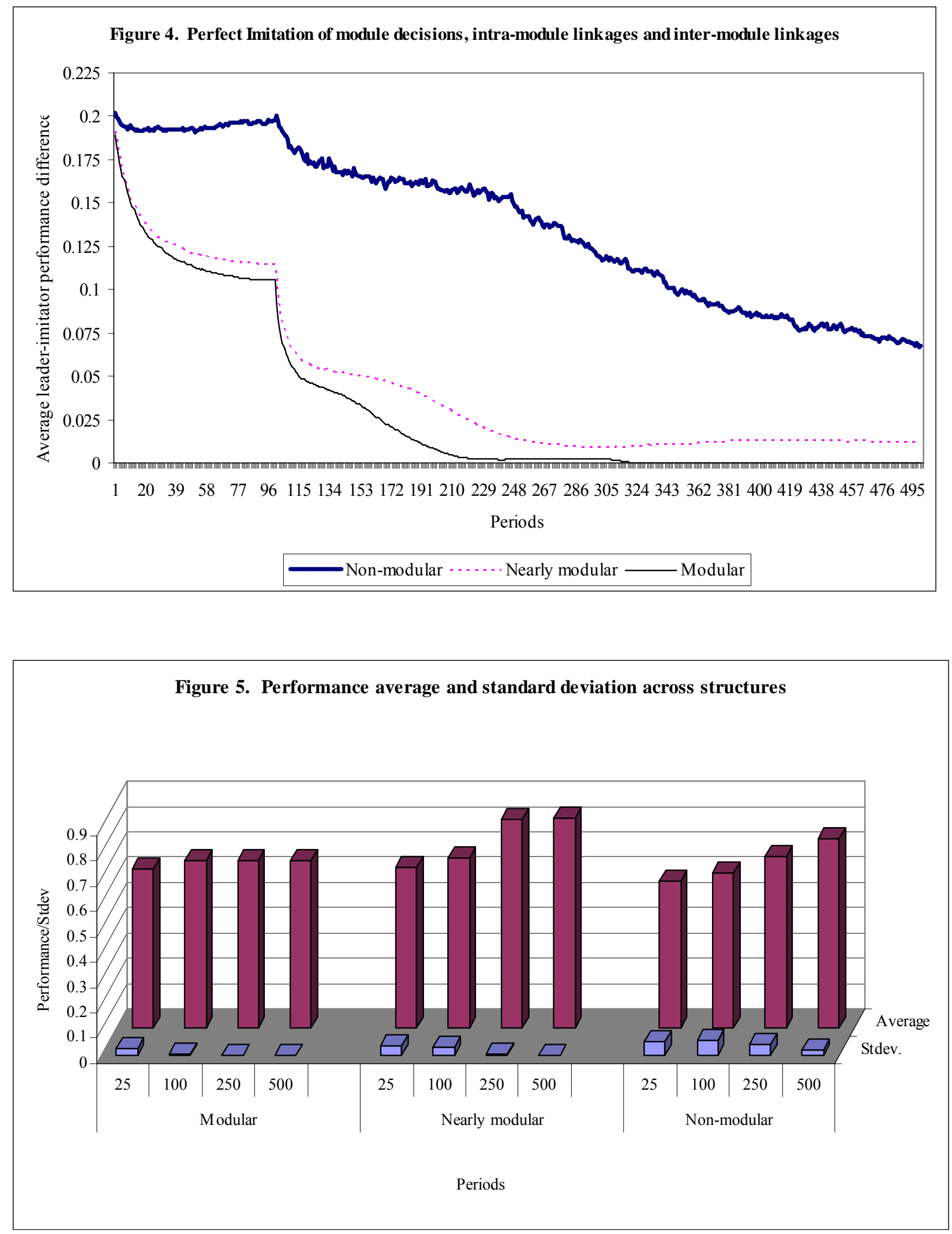

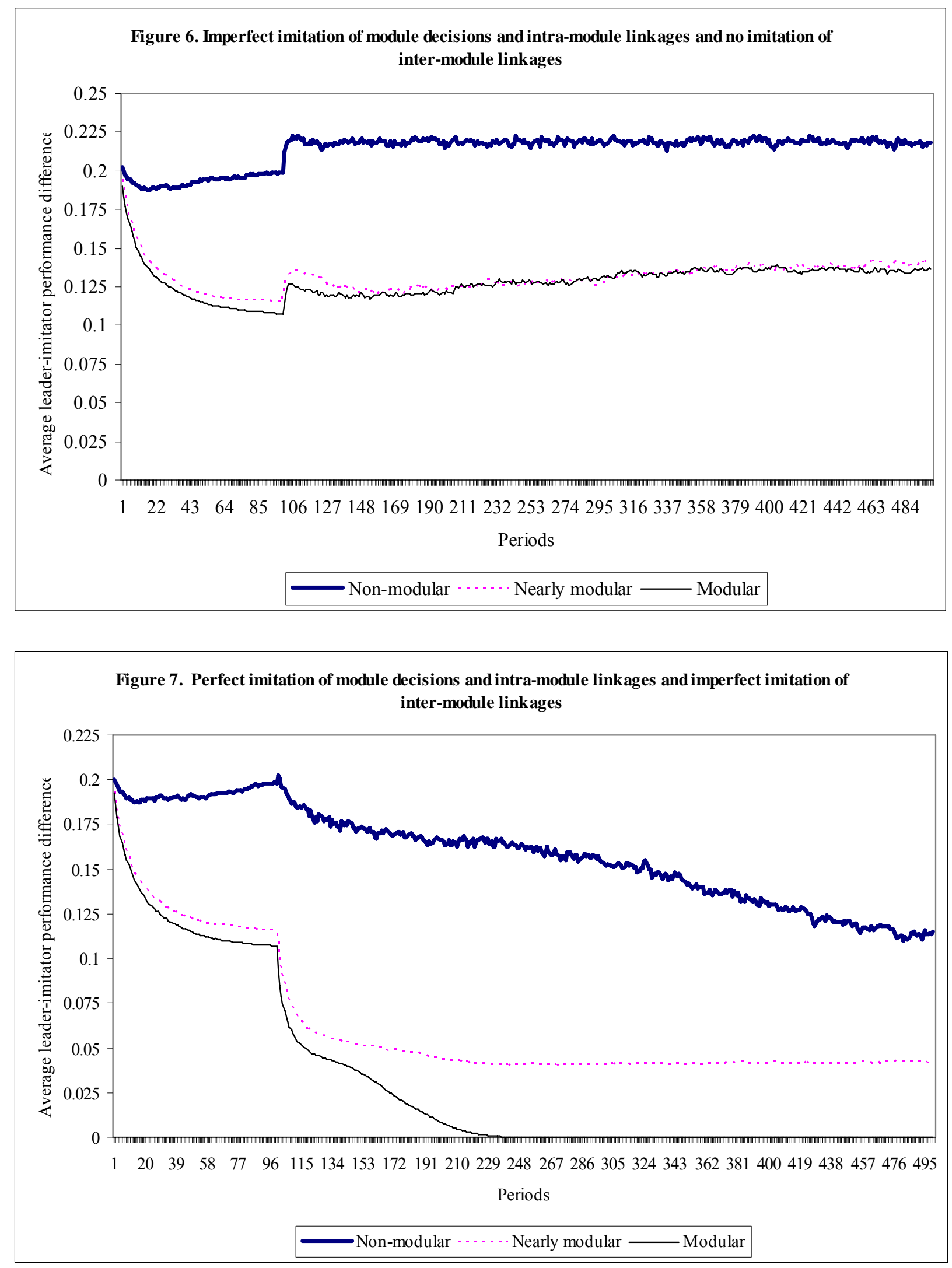


\section{Online Supplement}

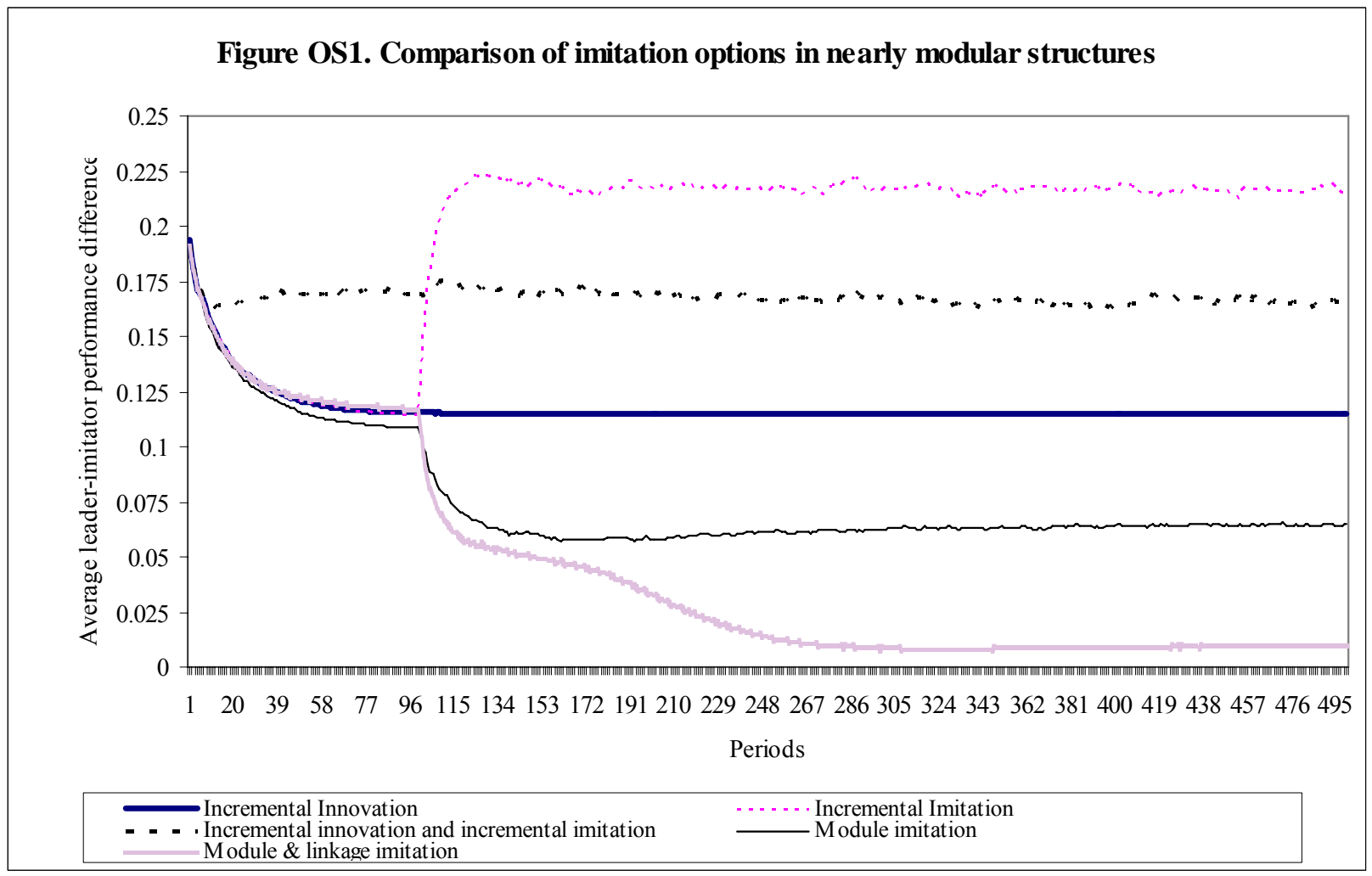

Notes: Figure OS1 evaluates the impact of alternative units of imitation including: (1) incremental innovation (single decision choices) only and no imitation; (2) Incremental imitation (single decision choices) only; (3) Incremental innovation and incremental imitation; (4) Module imitation; (5) module imitation and inter-module linkage imitation. The plot shows the average leader-imitator performance difference as the experiment progresses in each of the imitation regimes. The figure shows that in interdependent settings firms are better off not imitating at all (alternative 1) to engaging in incremental imitation (alternative 2) or incremental imitation in conjunction with incremental innovation (alternative 3). This is because imitation in interdependent systems destroys the value of incremental innovation and increases the average leader-imitator performance difference. In contrast, imitation alternatives (4) and (5) at the module level are superior to the status quo of no imitation. Thus, firms in interdependent settings, if they choose to engage in imitation, have a greater incentive to imitate modules and their linkages rather than single decision choices. 


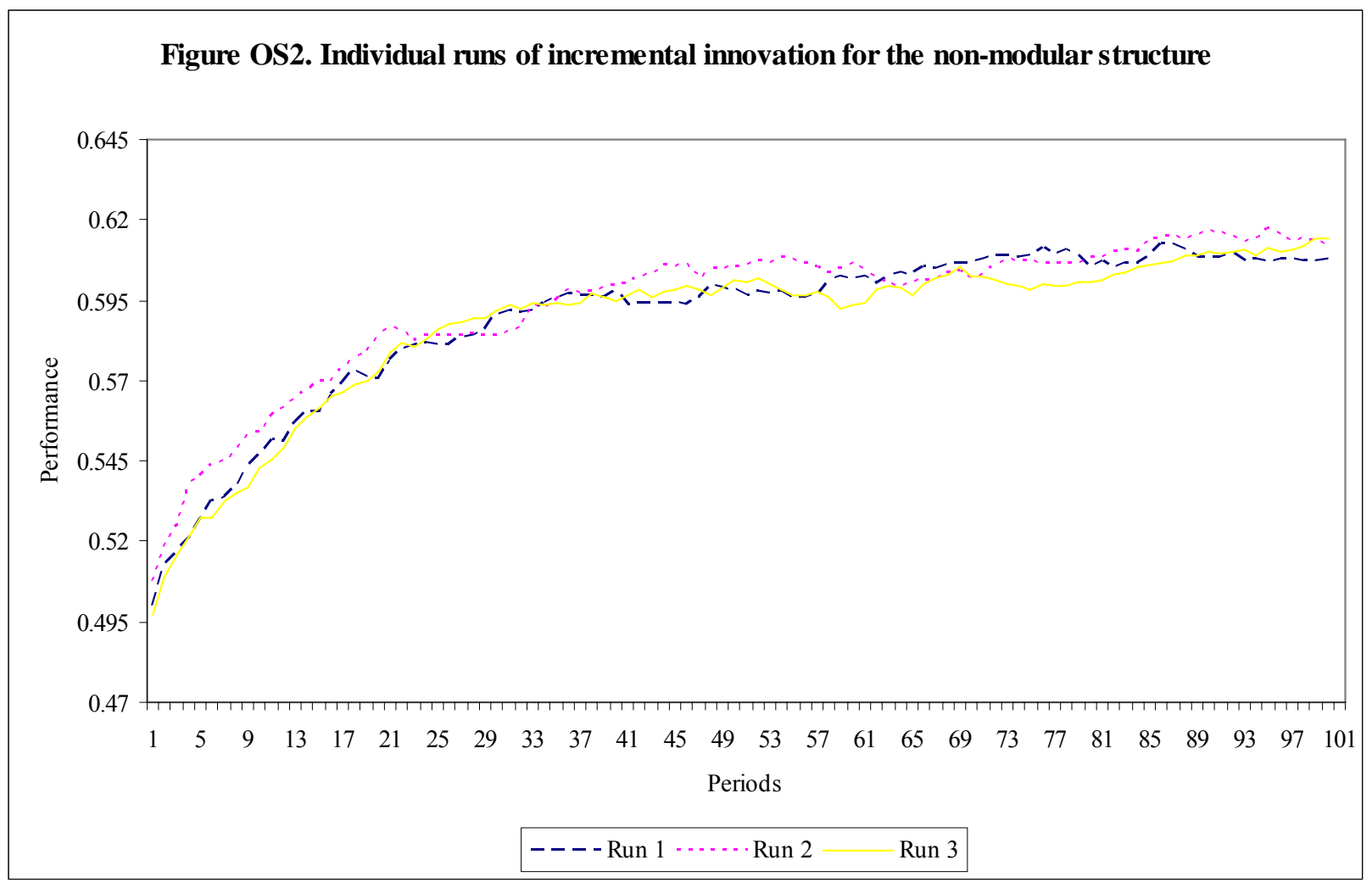

Notes: Figure OS2 shows three individual runs of incremental innovation in the non-modular structure. The single runs reveal the non-monotonicity of performance improvement that is a function of the inter-module interdependencies. As a result the non-modular structure does not reach a stable asymptote by the end of 100 periods. 


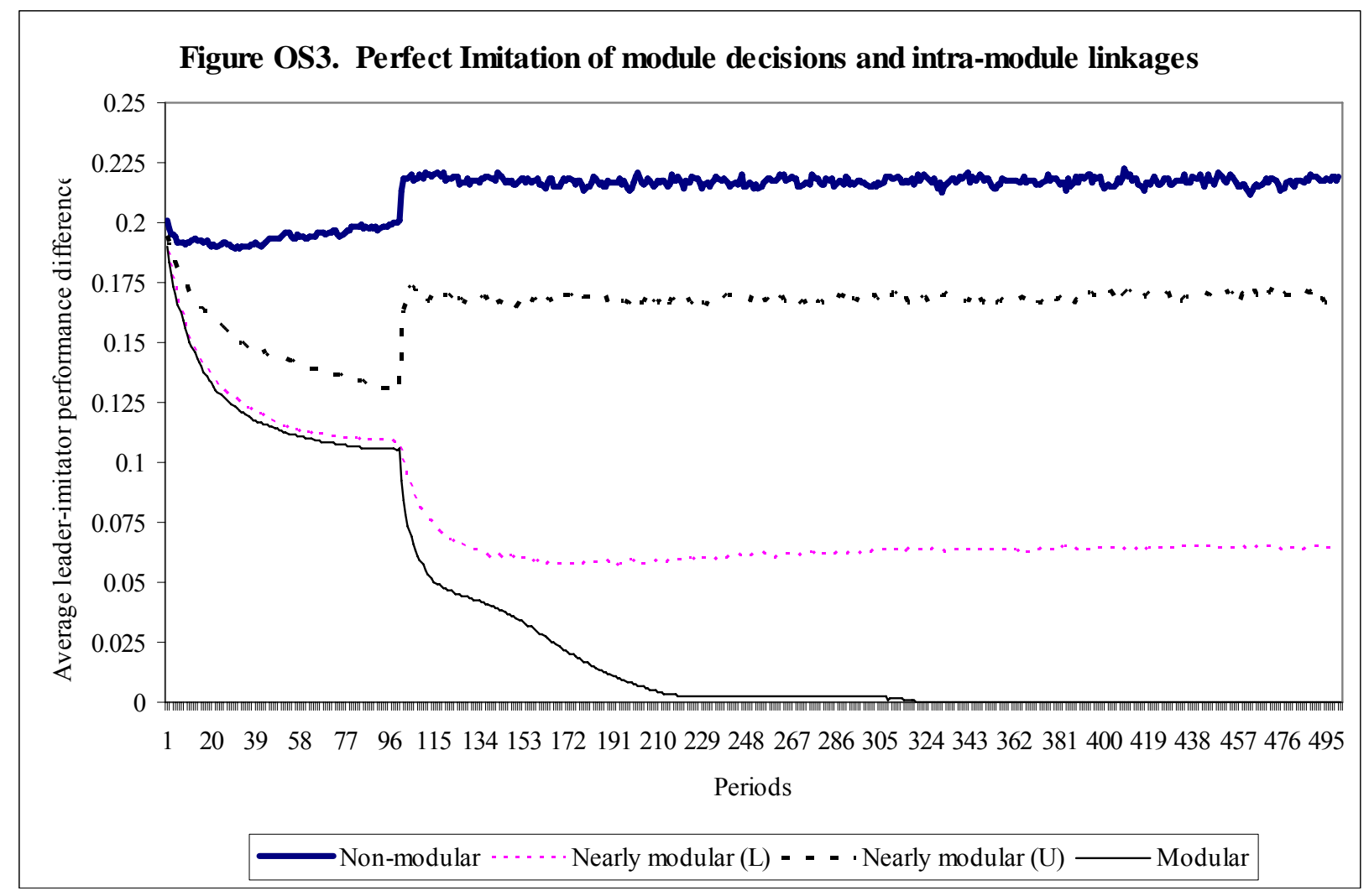

Notes: Figure OS3 is an augmented version of Figure 3 in the paper. We sought to parameterize nearly modular structures to capture the implications for the full range of such structures. We parameterized nearly modular structures by increasing the density of inter-module linkages and correspondingly reducing the density of intra-module linkages. We adopted a definition of nearly modular structures as those where the density of intra-module linkages are greater than the density of inter-module linkages. Thus, we added an upper bound on nearly modular structures and called it nearly modular $(\mathrm{U})$ and relabeled the nearly modular structure reported in the paper as nearly modular (L). From the figure above, we see that the benefits of imitation deterrence are increasing gradually in the extent of intermodule linkages and reach their maximum in the case of non-modular structures. 


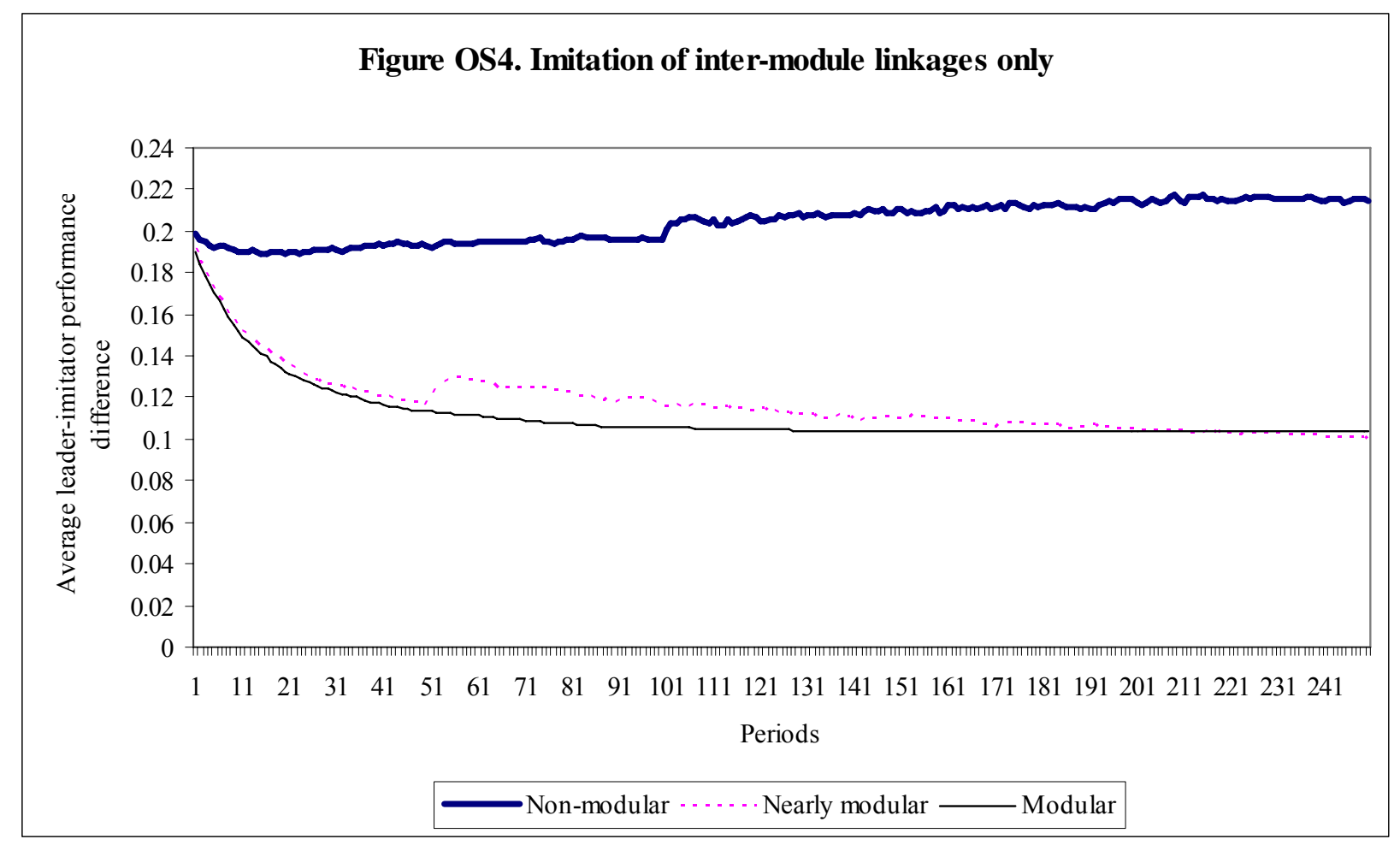

Notes: Figure OS4 shows the effects of imitation of inter-module linkages only. In the modular structure no imitation occurs since there are no inter-module linkages and thus the status quo of the leader-imitator performance difference is preserved. In the nearly modular structure, imitation of linkages initially worsens the performance of imitators before improving. Overall, linkage copying does little to bridge the gap between leaders and imitators. 


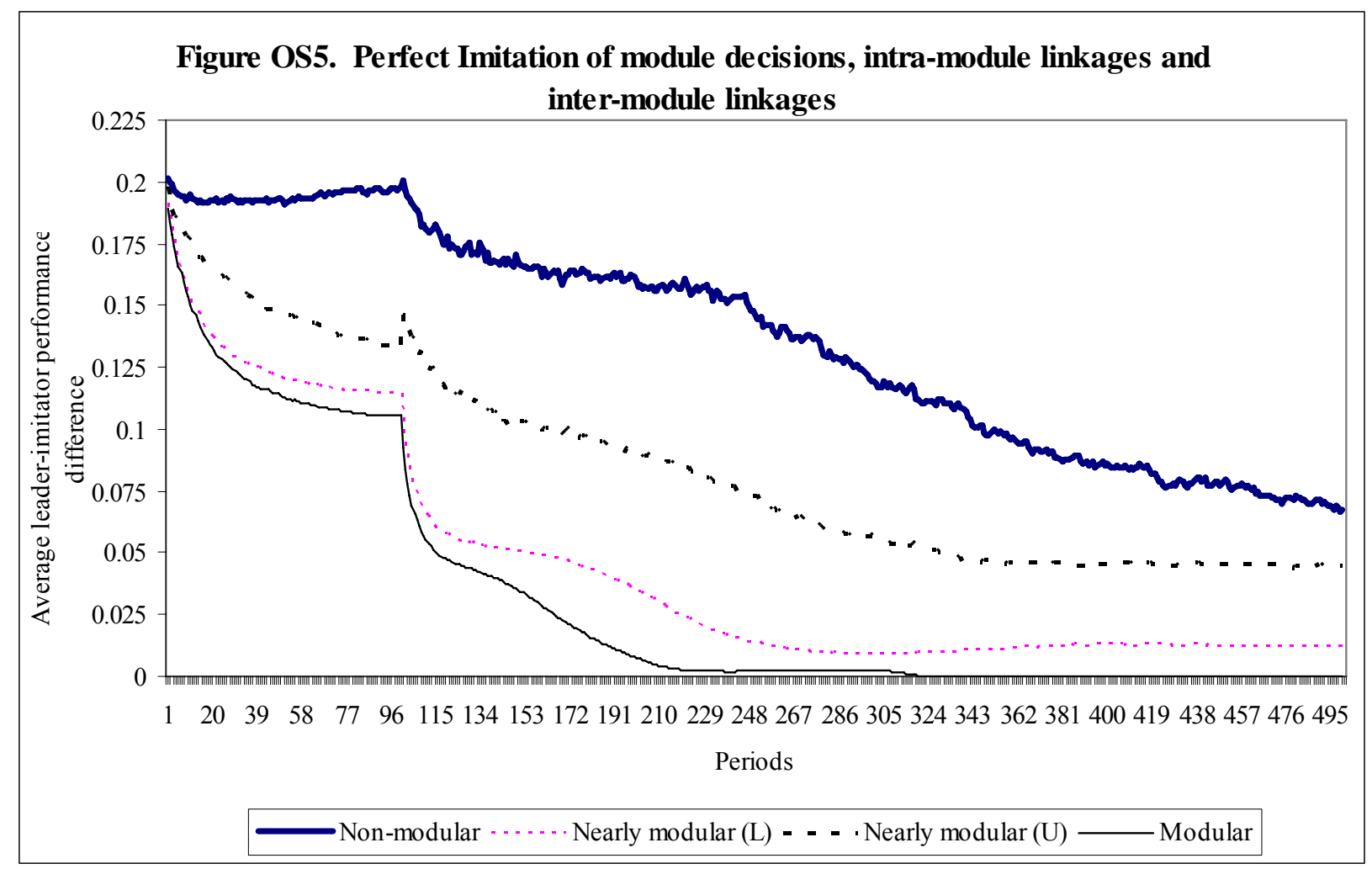

Notes: Figure OS5 is the augmented version of Figure 4 in the paper. We again parameterized the extent of nearly modular structures and added the results for nearly modular (U) structures (see description for Figure OS3). Once again the results show that the imitation deterrence potential of nearly modular structures is increasing in the extent of inter-module linkages and reaches its maximum in the case of the non-modular structures. Furthermore, the imitation deterrence potential remains substantial even in the extreme case of imitators being able to copy module decisions, intra-module linkages, and inter-module linkages. 


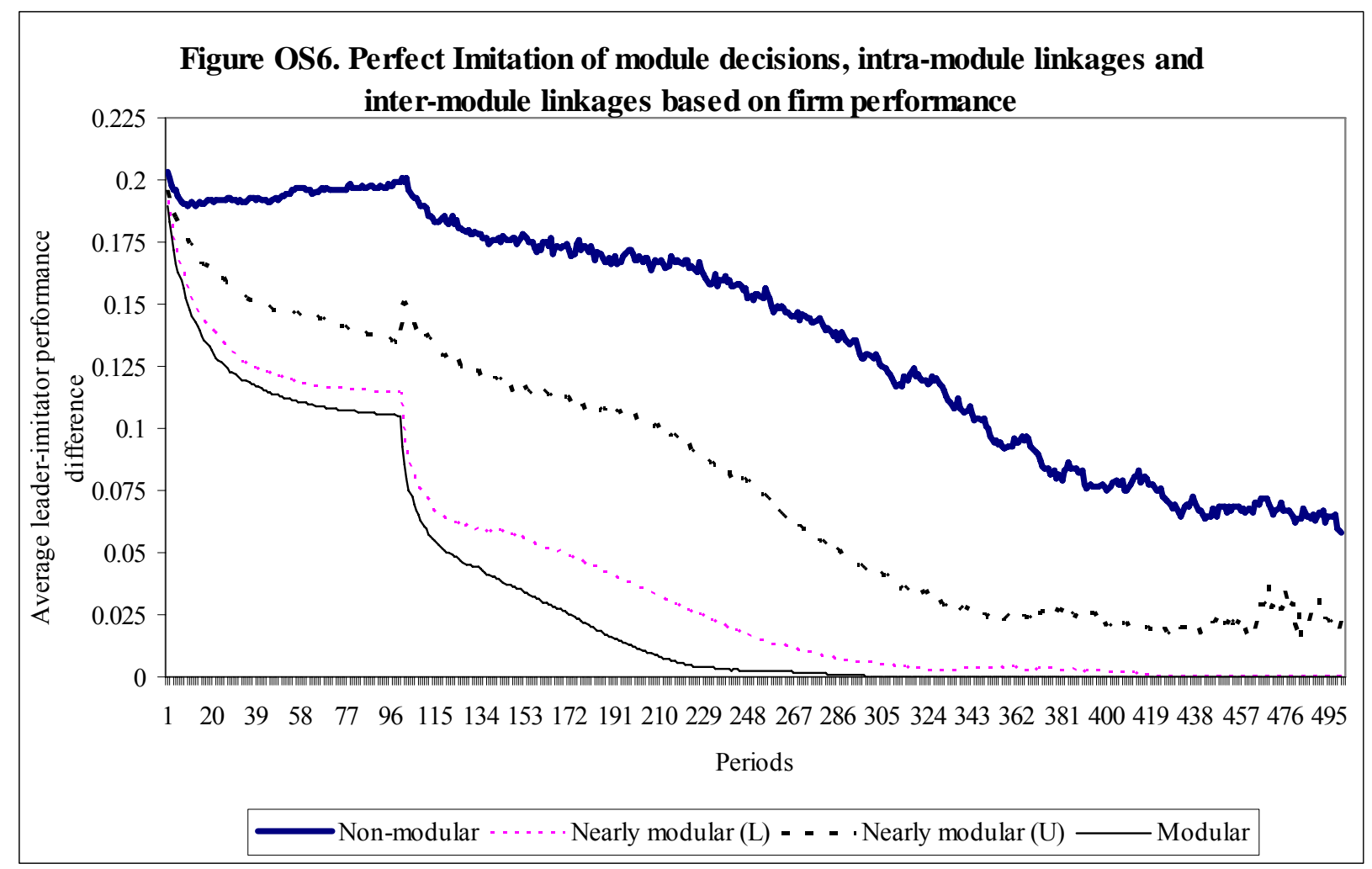

Notes: Figure OS6 implements the perfect imitation of module decisions, intra-module linkages and inter-module linkages based on superior firm performance rather than superior module performance. This is akin to imitating randomly chosen modules of high-performing firms. As seen from the figure, this regime diminishes much of the performance differences between leaders and imitators. In the nearly modular (L) structures the average leader-imitator performance difference goes to zero by period 431 in contrast to period 323 in the case of modular structure. The performance difference in the nearly modular $(\mathrm{U})$ and non-modular structures do not go to zero by the $500^{\text {th }}$ period but is likely if we run the model for a longer duration. Nonetheless, the relative ordering of the three structures in terms of its imitation deterrence potential remains robust. 


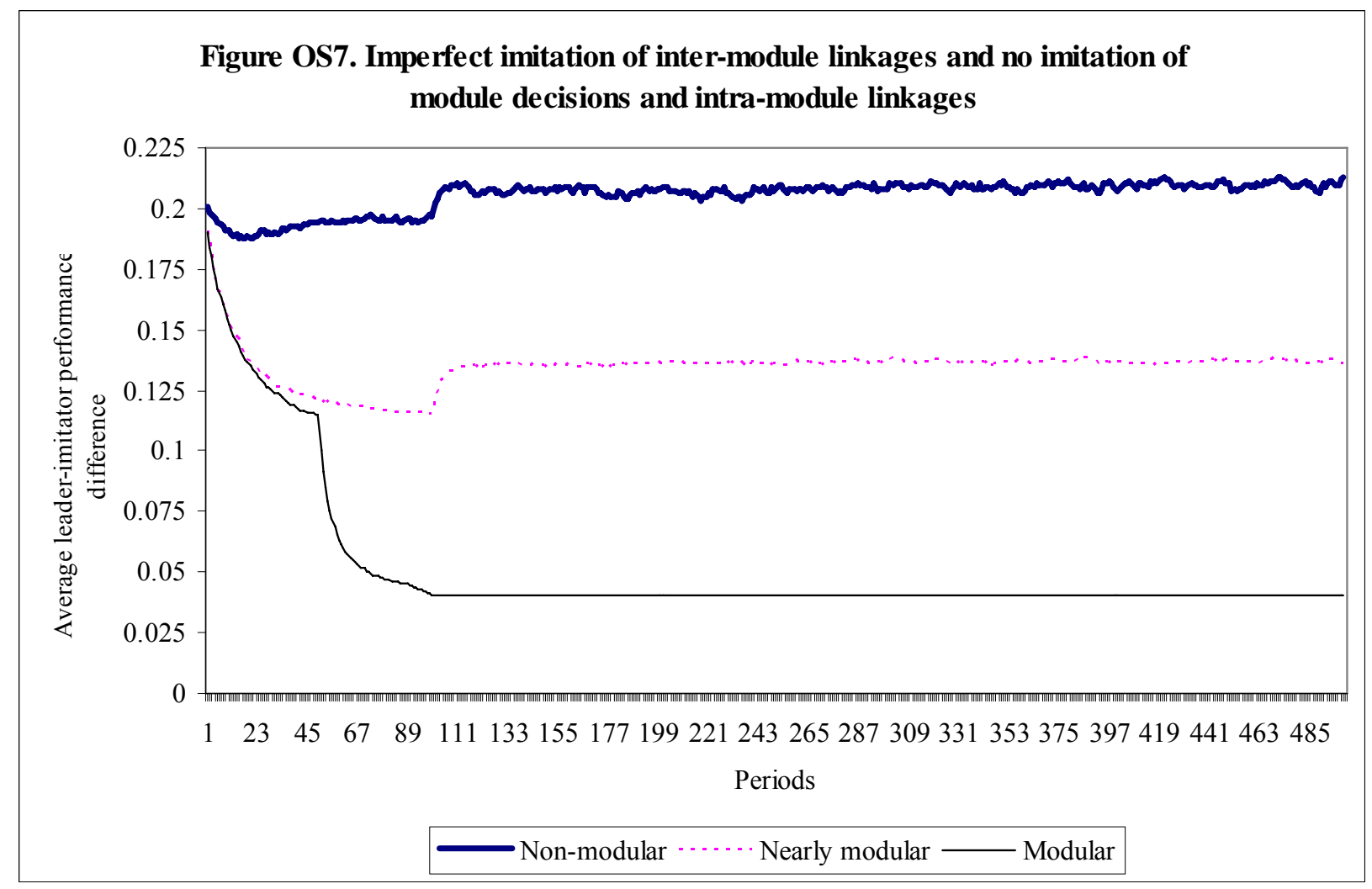

Notes: Figure OS7 shows the effect of imperfect imitation of only the inter-module linkages but not the module decisions and intra-module linkages. The modular structure maintains the status quo in the performance difference between leaders and imitators since no imitation occurs because there are no inter-module linkages. In the nearly modular and non-modular structures imperfect imitation of linkages alone hurts the performance of imitators suggesting that they may be better off not imitating at all. This again confirms the relative ordering of the three structures in terms of their imitation deterrence potential. 


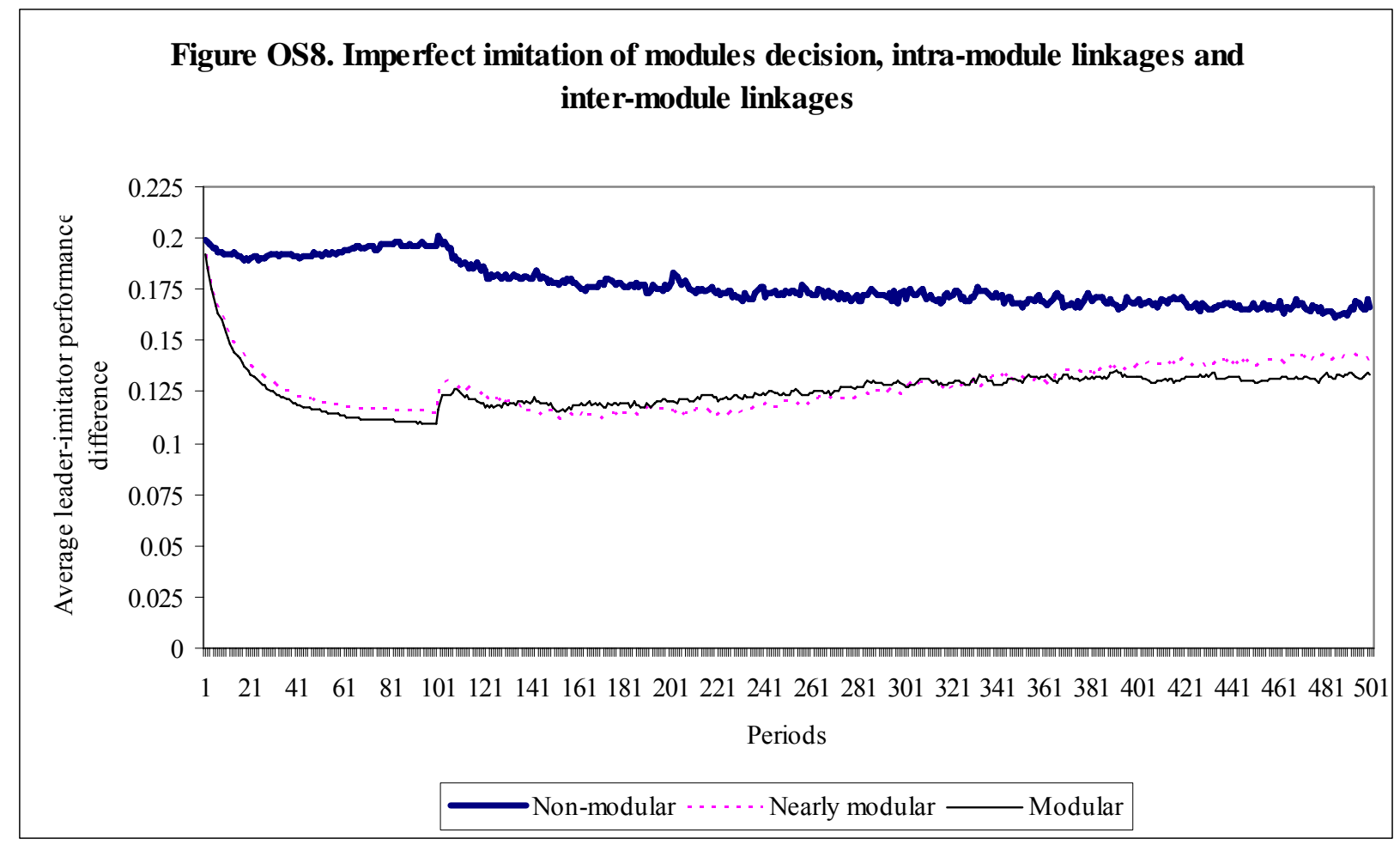

Notes: Figure OS8 shows the imperfect imitation of module decision, intra-module linkages, and intermodule linkages. These results show that the imitation deterrence of the three structures is largely similar when imitation is imperfect. Thus, the extent of imperfections in imitation acts as a substitute for design complexity. In other words, if imitation is expected to be highly imperfect, design complexity is not a discriminating instrument for imitation deterrence. 